\title{
NULL HYPERSURFACES EVOLVED BY THEIR MEAN CURVATURE IN A LORENTZIAN MANIFOLD
}

\author{
SAMUEL SSEKAJJA ${ }^{1}$, FORTUNÉ MASSAMBA ${ }^{2}$
}

\begin{abstract}
We use null isometric immersions to introduce time-dependent null hypersurfaces, in a Lorentzian manifold, evolving in the direction of their mean curvature vector (a vector transversal to the null hypersurface). We prove an existence result for such hypersurfaces in a short-time interval. Then, we discuss the evolution of some induced geometric objects. Consequently, we prove under certain geometric conditions that some of the above objects will blow-up in finite time. Also, several examples are given to illustrate the main ideas.
\end{abstract}

\section{Introduction}

Flow of Riemannian hypersurfaces by functions of their mean curvatures (for example; mean curvature flow, inverse mean curvature flow, and many more) has been an interesting area of research in the past 30 years. Numerous results have been obtained for these hypersurfaces, in the Riemannian case, for instance [15] shows that inverse mean curvature evolution can be used to relate the size of a black hole and its total energy. On the other hand, G. Huisken [14] showed that the solution to mean curvature evolution with some specified initial data remains smooth, compact and convex until it shrinks to a "round point" in a finite time; that is, the asymptotic shape of evolving hypersurface just before it disappears is a sphere. Gage and Hamilton [11] used curve shortening flow to prove isoperimetric inequality for convex planar domains, and Andrews [2] used affine mean curvature flow to prove affine isoperimetric inequalities. More results and details on Riemannian mean curvature evolution can be found in the above mentioned papers and references therein.

Lightlike hypersurfaces are closely linked to black hole theory and the study of trapped surfaces. In fact, it has been shown in [7] that black hole

2010 Mathematics Subject Classification. Primary 53C25; Secondary 53C40, 53C50.

Key words and phrases. Null hypersurfaces;Null mean curvature flow; Maximum principle. 
horizons can be represented by null hypersurfaces. The classical approach to the above phenomena has been the use of expansion scalars (null mean curvature) from null geodesics, via Raychaudhuri's equation (see [8] for details). Literature shows that our universe is expanding in time. This can be supported by the fact that very distant stars appear as redshifts. By definition, a red-shift is a change in color when an object that emits light is moving away from the observer [8]. It is called a redshift because the shifts are towards the red part of the spectrum. Heavenly bodies like stars are moving away from us radially, so we say that at one time the universe was smaller. Going back far enough in time, it is evident from above explanation that the universe may have been something the size of an atom; what we call the Primordial Atom [8]. Along with the expanding universe, since the black holes are surrounded by a local mass distribution and expand by the inflow of galactic debris as well as electromagnetic and gravitational radiation, their area increase in a given physical situation. Hence black holes are also expanding in time. Therefore, although the classical isolated black holes have been extensively studied (see [8] and references therein), they do not represent a realistic model in the context of an expansing universe. To address this issue of representing expanding black holes, Ashtekar-Krishnan [1, p. 268] introduced a concept of dynamical horizons which are a special type of spacelike hypersurfaces of a spacetime whose asymptotic states are the isolated horizons.

Motivated by the fact our Universe (and its constituents) is changing with time, we introduce a geometric evolution of null hypersurfaces a long their null mean curvature. In particular, this kind of evolution has a potential to explain, among other applications, the idea of trapped surfaces (hence, the study of black holes since trapped surfaces are linked to black holes). To the best of our understanding, this is the first piece of work in this direction on null hypersurfaces in Lorentzian manifold, despite the fact that; the study of null hypersurfaces started long time by Duggal-Bejancu [7] and Kupeli [20], and up-to-date results may be found in the books $[7,8]$. This motivated other researchers to investigate the geometry of null submanifolds, see for example $[3,4,6,16,18]$ among others.

The paper is arranged as follows; In Section 2 we quote some basic geometric tools necessary for the development of other sections. In Section 3, we define the concept of null Mean Curvature Flow (MCF) for null hypersurfaces, using null isometric immersions (see Definition 3.2), and give some 
supporting physical example. Section 4 is devoted to existence results and geometry of induced objects on the evolving hypersurface.

\section{Preliminaries}

Consider an $(n+1)$-dimensional null hypersurface $(M, g), n \geq 2$, of a $(n+2)$-dimensional Lorentzian manifold $(\bar{M}, \bar{g})$, where $g$ is a degenerate metric on $M$, induced by the Lorentzian metric $\bar{g}$ of $\bar{M}$. In the null hypersurface case, basic differences occur mainly due to the fact that the normal vector bundle $T M^{\perp}$ is the same as the null tangent bundle along a non-zero differentiable radical distribution $\operatorname{Rad} T M$ of $M$, defined by $\operatorname{Rad} T_{p} M=T_{p} M^{\perp}=\left\{E_{p} \in T_{p} M: g\left(E_{p}, X\right)=0, \quad \forall X \in T_{p} M\right\}$, where $\operatorname{dim}(\operatorname{Rad} T M)=1$. There exists a Riemannian screen distribution [7], denoted $S(T M)$, on $M$ which is complementary to the radical distribution such that we have the orthogonal direct sum $T M=T M^{\perp} \perp S(T M)$. Throughout this paper, $\Gamma(\Xi)$ will denote the $\mathcal{F}(M)$-module of differentiable sections of a vector bundle $\Xi$. The manifolds we consider are supposed to be paracompact, smooth and connected. Therefore, the existence of $S(T M)$ is secured. However, in general, $S(T M)$ is not canonical (thus not unique) and the null geometry depends on its choice. But it is known $[7,8]$ that $S(T M)$ is canonically isomorphic to the fact bundle $T M / T M^{\perp}$ considered by Kupeli [20].

From [7, p. 79, Theorem 1.1], we know that for a screen distribution $S(T M)$ on $M$ there exists a unique vector bundle $\operatorname{tr}(T M)$ such that for any non-zero local normal section $E \in \Gamma\left(\left.\operatorname{Rad} T M\right|_{\mathcal{U}}\right)$ on $\mathcal{U} \subset M$ there exists a unique section $N$ of $\left.\operatorname{tr}(T M)\right|_{\mathcal{U}}$ satisfying $\bar{g}(E, N)=1, \bar{g}(N, Z)=0$, for all $Z \in \Gamma\left(\left.S(T M)\right|_{\mathcal{U}}\right)$. Then, we have the decomposition $\left.T \bar{M}\right|_{M}=T M \oplus$ $\operatorname{tr}(T M)$.

Let $\nabla$ and $\nabla^{*}$ denote the induced connections on $M$ and $S(T M)$, respectively, and $P$ be the projection of $T M$ onto $S(T M)$, then the local Gauss-Weingarten equations of $M$ and $S(T M)$ are the following [7]

$$
\begin{gathered}
\bar{\nabla}_{X} Y=\nabla_{X} Y+h(X, Y)=\nabla_{X} Y+B(X, Y) N, \\
\bar{\nabla}_{X} N=-A_{N} X+\nabla_{X}^{t} N=-A_{N} X+\tau(X) N, \\
\nabla_{X} P Y=\nabla_{X}^{*} P Y+h^{*}(X, P Y)=\nabla_{X}^{*} P Y+C(X, P Y) E, \\
\nabla_{X} E=-A_{E}^{*} X+\nabla_{X}^{* t} E=-A_{E}^{*} X-\tau(X) E, \quad A_{E}^{*} E=0,
\end{gathered}
$$


for all $X, Y \in \Gamma(T M), E \in \Gamma\left(T M^{\perp}\right)$ and $N \in \Gamma(\operatorname{tr}(T M))$, where $\bar{\nabla}$ is the Levi-Civita connection on $\bar{M}$. In the above setting, $B$ is the local second fundamental form of $M$ and $C$ is the local second fundamental form on $S(T M) . A_{N}$ and $A_{E}^{*}$ are the shape operators on $T M$ and $S(T M)$ respectively, while $\tau$ is a 1-form on $T M$. The above shape operators are related to their local fundamental forms by

$$
\begin{aligned}
& g\left(A_{E}^{*} X, Y\right)=B(X, Y), \quad g\left(A_{N} X, P Y\right)=C(X, P Y), \\
& \bar{g}\left(A_{E}^{*} X, N\right)=0, \quad \bar{g}\left(A_{N} X, N\right)=0, \quad \forall X, Y \in \Gamma(T M) .
\end{aligned}
$$

From (2.6) we notice that $A_{E}^{*}$ and $A_{N}$ are both screen-valued operators. Let $\vartheta=\bar{g}(N, \cdot)$ be a 1 -form metrically equivalent to $N$ defined on $\bar{M}$. Take $\lambda=i^{*} \vartheta$ to be its restriction on $M$, where $i: M \rightarrow \bar{M}$ is the inclusion map. Then it is easy to show that

$$
\left(\nabla_{X} g\right)(Y, Z)=B(X, Y) \lambda(Z)+B(X, Z) \lambda(Y), \quad \forall X, Y, Z \in \Gamma(T M),
$$

which indicates that $\nabla$ is generally not a metric connection with respect to $g$. However, the induced connection $\nabla^{*}$ on $S(T M)$ is a metric connection. For more details about null hypersurfaces see the books [7] and [8].

The degeneracy of induced metric tensor $g$, on $M$, is associated with several challenges in the study of null geometry, for instance see the books [7] and [8]. Prior to these books, the author in [21] introduced a non-degenerate metric (called the associated mertic) on null hypersurfaces in 4-dimentional Lorentzian manifolds. This metric was extended to null hypersurfaces in $(n+2)$-dimensional Lorentzian manifold in [4] and used to define some geometric operators on null hypersurfaces. It has also been linked to null rigging techniques on null hypersurfaces, for example, see [10] and other references therein. More precisely, for a null hypersurface $(M, g)$ of a Lorentzian manifold $(\bar{M}, \bar{g})$, the associated metric $\widehat{g}$ is given by

$$
\widehat{g}(X, Y)=g(X, Y)+\lambda(X) \lambda(Y), \quad \forall X, Y \in \Gamma(T M) .
$$

The metric $\widehat{g}$ is invertible and its inverse, $g^{[\cdot, \cdot]}$, was called the pseudo-inverse of $g$ (see [4]). Also, observe that $\widehat{g}$ coincides with $g$ if the latter is nondegenerate. The metric $\widehat{g}$ has been used to define (on $M$ ) the usual operators such as gradient, divergence, D' Alambertian (see [4]), which one can not afford with the degenerate metric $g$. In case $\widehat{g}$ coincide with $g$ on $M$, we define the gradient $\nabla^{s} f$, Hessian $\operatorname{Hess}^{s}(f)$, and D' Alambertian $\Delta^{s} f$ of a smooth function $f$ on $\mathcal{U} \subset M$ with respect to the screen distribution $S(T M)$ 
as

$$
\begin{gathered}
\nabla^{s} f=g^{\alpha \beta} X_{\alpha}(f) X_{\beta}, \quad \operatorname{Hess}^{s}(f)=X_{\alpha}\left(X_{\beta}(f)\right)-\left(\nabla_{X_{\alpha}}^{*} X_{\beta}\right)(f) \\
\Delta^{s}(f)=\operatorname{tr}^{s}\left(\operatorname{Hess}^{s}(f)\right)=g^{\alpha \beta}\left(X_{\alpha}\left(X_{\beta}(f)\right)-\left(\nabla_{X_{\alpha}}^{*} X_{\beta}\right)(f)\right),
\end{gathered}
$$

where $\left\{X_{1}, \ldots, X_{n}\right\}$ is a basis of $S(T M)$ and $\operatorname{tr}^{s}(\cdot)$ denotes the trace with respect to $S(T M)$. Throughout this paper, we assume that $M$ carries the associated metric $\widehat{g}$ and $\operatorname{tr}(\cdot)$ will denote the trace over $M$ with respect to $\widehat{g}$.

Denote by $R$ and $\bar{R}$, the curvature tensors of $M$ and $\bar{M}$ respectively. Then, by (cf. [7], p. 94), we have

$$
\begin{aligned}
\bar{g}(\bar{R}(X, Y) Z, P W) & =g(R(X, Y) Z, P W)+B(X, Z) C(Y, P W) \\
& -B(Y, Z) C(X, P W), \quad \forall X, Y, Z, W \in \Gamma(T M) .
\end{aligned}
$$

In the sequel, we shall make use of the following convention on the range of indices: $1 \leq \alpha, \beta, \gamma, \mu, \sigma \leq n, 0 \leq a, b, c \leq n$ and $0 \leq i, j, k \leq n+1$.

\section{Null mean curvature flow}

The concept of null hypersurfaces (and generally, submanifolds) can be presented alternatively by using a special isometric immersion [7]. Let $(M, g)$ be a 1-null manifold of dimension $n+1$ and index $q-1, n>0$ and $q>0$. Let $S(T M)$ be the screen distribution of $M$ as in the previous section. Suppose there exist a vector bundle $\Lambda$ of rank 1 over $M$ such that $T \bar{M}=T M \oplus \Lambda$ is a semi-Riemannian vector bundle with a semi-Riemannian metric $\bar{g}$ satisfying

$$
\left(C_{1}\right) \quad \bar{g}(X, Y)=g(X, Y), \quad \bar{g}(Z, V)=\bar{g}\left(V, V^{\prime}\right)=0,
$$

for any $X, Y \in \Gamma(T M), Z \in \Gamma(S(T M))$ and $V, V^{\prime} \in \Gamma(\Lambda)$. Since $\bar{g}$ is nondegenerate on $E$, it follows that $\bar{g}(U, V) \neq 0$, for any non-zero vector fields $U \in \Gamma(\operatorname{Rad} T M)$ and $V \in \Gamma(\Lambda)$. Suppose there exists a torsion-free linear connection $\nabla^{\prime}$ on $\mathrm{M}$ and a linear connection $\nabla^{\text {tr }}$ on $\Lambda$ satisfying

$$
\left(C_{2}\right) \quad \bar{g}\left(\nabla_{X}^{\prime} U, V\right)+\bar{g}\left(U, \nabla_{X}^{t r} V\right)=X(\bar{g}(U, V)),
$$

For any $X \in \Gamma(T M), U \in \Gamma(\operatorname{Rad} T M)$ and $V \in \Gamma(\Lambda)$. As

$$
T M=S(T M) \perp \operatorname{Rad} T M,
$$

we the following decompositions

$$
\begin{aligned}
\nabla_{X}^{\prime} P Y & =\nabla_{X}^{*^{\prime}} P Y+h^{*^{\prime}}(X, P Y), \quad \forall X, Y \in \Gamma(T M), \\
\nabla_{X}^{\prime} U & =-A_{U}^{*^{\prime}} X+\nabla_{X}^{* t r} U, \quad \forall X \in \Gamma(T M), \quad U \in \Gamma(\operatorname{Rad} T M),
\end{aligned}
$$


where $P$ is the projection morphism of $T M$ onto $S(T M),\left\{\nabla_{X}^{*^{\prime}} P Y, A_{U}^{*^{\prime}} X\right\}$ and $\left\{h^{*^{\prime}}(X, P Y), \nabla_{X}^{* t r} U\right\}$ belongs to $S(T M)$ and $\operatorname{Rad} T M$, respectively. It follows that $\nabla^{*^{\prime}}$ and $\nabla^{* t r}$ are linear connections on the vector bundles $S(T M)$ and $\operatorname{Rad} T M$, respectively. On the other hand, $h^{*^{\prime}}$ and $A^{*^{\prime}}$ are $\mathcal{F}(M)$-bilinear forms on $\Gamma(T M) \times \Gamma(S(T M))$ and on $\Gamma(\operatorname{Rad} T M) \times \Gamma(T M)$, respectively. Besides, we suppose that the following conditions hold

$\left(C_{3}\right) \quad A_{U}^{*^{\prime}} U=0, \quad g\left(A_{U}^{*^{\prime}} X, Y\right)=g\left(X, A_{U}^{*^{\prime}} Y\right)$,

$\left(C_{4}\right) \quad\left(\nabla_{X}^{\prime} g\right)(P Y, P Z)=\left(\nabla_{X}^{\prime} g\right)(U, U)=0, \quad\left(\nabla_{X}^{\prime} g\right)(P Y, U)=g\left(A_{U}^{*^{\prime}} X, P Y\right)$,

$\left(C_{5}\right)\left(\nabla_{X}^{\prime} A^{*^{\prime}}\right)(U, Y)=\left(\nabla_{Y}^{\prime} A^{*^{\prime}}\right)(U, X), \forall X, Y, Z \in \Gamma(T M), U \in \Gamma(\operatorname{Rad} T M)$,

where $\left(\nabla_{X}^{\prime} A^{*^{\prime}}\right)(U, Y)=\nabla_{X}^{\prime} A_{U}^{*^{\prime}} Y-A_{\nabla_{X}^{* t r} U}^{*^{\prime}} Y-A_{U}^{*^{\prime}} \nabla_{X}^{\prime} Y$. Denote by $R^{\prime}$ the curvature tensor of $\nabla^{\prime}$ and further suppose conditions:

$$
\begin{aligned}
& \left(C_{6}\right) \quad g\left(R^{\prime}(X, Y) Z, P W\right)=g\left(A_{h^{*^{\prime}}(X, P W)}^{*^{\prime}} Y, Z\right)-g\left(A_{h^{*^{\prime}}(Y, P W)}^{*^{\prime}} X, Z\right), \\
& \left(C_{7}\right) \quad \bar{g}\left(R^{\prime}(X, Y) Z, V\right)=0, \forall X, Y, Z, W \in \Gamma(T M), V \in \Gamma(\Lambda) .
\end{aligned}
$$

Let $(M, g, S(T M))$ be a 1-null simply connected $(n+1)$-dimensional manifold of index $q-1$, endowed with the vector bundle $\Lambda$ and geometric objects $\bar{g}$, $\nabla^{\prime}, \nabla^{t r}, h^{*^{\prime}}$ and $A^{*^{\prime}}$ satisfying conditions $\left(C_{1}\right)-\left(C_{7}\right)$. Then there exists a null isometric immersion $F: M^{n+1} \longrightarrow \bar{M}^{n+2}[7$, Theorem 4.1] satisfying

$$
g(X, Y)=\bar{g}\left(F_{*} X, F_{*} Y\right), \quad \forall X, Y \in \Gamma(T M),
$$

and a vector bundle isomorphism $\bar{F}: \operatorname{tr}(T M) \longrightarrow \operatorname{tr}(T F(M))$ such that

$$
\begin{gathered}
F_{*}\left(\nabla_{X}^{\prime} Y\right)=\nabla_{F_{*} X} F_{*} Y, \quad F_{*}\left(A_{U}^{*^{\prime}} X\right)=A_{F_{*} U}^{*} F_{*} X, \\
F_{*}\left(h^{*^{\prime}}(X, P Y)\right)=h^{*}\left(F_{*} X, F_{*} P Y\right), \quad \bar{F}\left(\nabla_{X}^{t r} V\right)=\nabla_{X}^{t} \bar{F} V,
\end{gathered}
$$

$\forall X, Y \in \Gamma(T M), U \in \Gamma(\operatorname{Rad} T M)$ and $V \in \Gamma(\operatorname{tr}(T M))$, where $\operatorname{tr}(T F(M))$ is the null transversal vector bundle of $F(M)$ with respect to $F_{*} S(T M)$, and $\nabla, \nabla^{t}, h^{*}, A^{*}$ are the geometric objects induced on $F(M)$ with respect to the immersion $F$. Certainly, $F(M)$ is nothing but a 1-null submanifold of $\bar{M}^{n+2}$ and $F$ preserves both the radical and screen distribution, that is; $F_{*}$ respectively maps the radical subspace and the screen of the domain to that of the base (see details in [7, p. 104]). More precisely,

$$
\operatorname{Rad} T F(M)=F_{*} \operatorname{Rad} T M, \quad S(T F(M))=F_{*} S(T M) .
$$

Next, suppose that $F(M)$ is a null hypersurface as described above. By the method of [7], null mean curvature vector $\mathrm{H}$ of $F(M)$ at $p \in M$ is a 
smooth vector field transversal to $F(M)$ and given by

$$
\mathrm{H}=\operatorname{tr}^{S}(h)=\operatorname{tr}^{s}(B) N=\mathcal{S} N
$$

where $N \in \Gamma(\operatorname{tr}(F T M))$ and $\mathcal{S}:=\operatorname{tr}^{S}(B)=\operatorname{tr}^{S}\left(A_{E}^{*}\right)$. The function $\mathcal{S}$ is called the null mean curvature of $F(M)$. Thus, $F(M)$ will be said to have constant mean curvature if $\mathcal{S}$ is constant.

In the following example, we calculate $\mathcal{S}$ for a Monge null hypersurface.

Example 3.1 (Null Monge hypersurface of $\mathbb{R}_{1}^{n+2}$ ). Consider a non-zero smooth function $G: \Sigma \longrightarrow \mathbb{R}$, where $\Sigma$ is an open set of $\mathbb{R}_{1}^{n+2}$. It is well-known $[7,8]$ that $M=\left\{\left(x_{0}, \ldots, x_{n+1}\right) \in \mathbb{R}_{1}^{n+2}: x_{0}=G\left(x_{1}, \ldots, x_{n+1}\right)\right\}$ is a Monge hypersurface. Moreover, it is easy to check that $M$ is null hypersurface if and only if $G$ is a solution of the partial differential equation $\sum_{i=1}^{n+1}\left(G_{x_{i}}^{\prime}\right)^{2}=1$. Then $\operatorname{Rad} T M$ and $\operatorname{tr}(T M)$ are respectively spanned by the global vector fields $E=\frac{\partial}{\partial x_{0}}+\sum_{i=1}^{n+1} G_{x_{i}}^{\prime} \frac{\partial}{\partial x_{i}}$ and $N=\frac{1}{2}\left(-\frac{\partial}{\partial x_{0}}+\sum_{i=1}^{n+1} G_{x_{i}}^{\prime} \frac{\partial}{\partial x_{i}}\right)$. The corresponding screen distribution is given by $\left\{Z_{1}, \ldots, Z_{n}\right\}$, where $Z_{\alpha}=$ $G_{x_{n+1}}^{\prime} \frac{\partial}{\partial x_{\alpha}}-G_{x_{\alpha}}^{\prime} \frac{\partial}{\partial x_{n+1}}$, for $\alpha \in\{1, \ldots, n\}$. Differentiating $\sum_{i=1}^{n+1}\left(G_{x_{i}}^{\prime}\right)^{2}=1$ we get $\sum_{i=1}^{n+1} G_{x_{i}}^{\prime} G_{x_{i} x_{j}}^{\prime \prime}=0$, for all $i, j \in\{1, \ldots, n+1\}$. Thus, $\bar{\nabla}_{E} E=$ $\sum_{j=1}^{n+1} \sum_{i=1}^{n+1} G_{x_{i}}^{\prime} G_{x_{i} x_{j}}^{\prime \prime} \frac{\partial}{\partial x_{j}}=0$. Also, by simple calculations we have

$$
\bar{\nabla}_{Z_{\alpha}} E=\sum_{i=1}^{n+1}\left(G_{x_{n+1}}^{\prime} G_{x_{\alpha} x_{i}}^{\prime \prime}-G_{x_{\alpha}}^{\prime} G_{x_{n+1} x_{i}}^{\prime \prime}\right) \frac{\partial}{\partial x_{i}}
$$

Considering the fact that $\frac{\partial}{\partial x_{\beta}}=\left(G_{x_{n+1}}^{\prime}\right)^{-1}\left(Z_{\beta}-G_{x_{\beta}}^{\prime} \frac{\partial}{\partial x_{n+1}}\right),(3.9)$ simplifies as follows

$$
\begin{aligned}
\bar{\nabla}_{Z_{\alpha}} E & =\sum_{\beta=1}^{n}\left(G_{x_{\alpha} x_{\beta}}^{\prime \prime}-\left(G_{x_{n+1}}^{\prime}\right)^{-1} G_{x_{\alpha}}^{\prime} G_{x_{n+1} x_{\beta}}^{\prime \prime}\right) Z_{\beta} \\
& +\sum_{\beta=1}^{n}\left(G_{x_{\alpha} x_{\beta}}^{\prime \prime} G_{x_{\beta}}^{\prime}-\left(G_{x_{n+1}}^{\prime}\right)^{-1} G_{x_{\beta}}^{\prime} G_{x_{n+1} x_{\beta}}^{\prime \prime}\right) \frac{\partial}{\partial x_{n+1}} \\
& +\left(G_{x_{n+1}}^{\prime} G_{x_{\alpha} x_{n+1}}^{\prime \prime}-G_{x_{\alpha}}^{\prime} G_{x_{n+1} x_{n+1}}^{\prime \prime}\right) \frac{\partial}{\partial x_{n+1}}
\end{aligned}
$$

Using the fact that $\sum_{\beta=1}^{n}\left(G_{x_{\alpha} x_{\beta}}^{\prime \prime}\right)+G_{x_{n+1}}^{\prime} G_{x_{\alpha} x_{n+1}}^{\prime \prime}=0$ in (3.10) we deduce that

$$
\bar{\nabla}_{Z_{\alpha}} E=\sum_{\beta=1}^{n}\left(G_{x_{\alpha} x_{\beta}}^{\prime \prime}-\left(G_{x_{n+1}}^{\prime}\right)^{-1} G_{x_{\alpha}}^{\prime} G_{x_{n+1} x_{\beta}}^{\prime \prime}\right) Z_{\beta}
$$


From (3.11) and $\sum_{i=1}^{n+1} G_{x_{i}}^{\prime} G_{x_{i} x_{j}}^{\prime \prime}=0$ it follows that the mean curvature function $\mathcal{S}$ is given by

$$
\mathcal{S}=\sum_{\alpha=1}^{n}\left(G_{x_{\alpha} x_{\alpha}}^{\prime \prime}-\left(G_{x_{n+1}}^{\prime}\right)^{-1} G_{x_{\alpha}}^{\prime} G_{x_{n+1} x_{\alpha}}^{\prime \prime}\right)=\sum_{i=1}^{n+1} G_{x_{i} x_{i}}^{\prime \prime} .
$$

3.1. Velocity on $M$. In order to introduce mean curvature flow for null hypersurfaces we need to recall some basic facts about velocity in manifolds from $[19$, p. $10-11]$. To that end, let $\sigma: I \longrightarrow \mathbb{R}$ be a smooth curve in a manifold $M$, where $I$ is an open interval of $\mathbb{R}$. In fact, $I$ has a coordinate system consisting of the identity map $v$ of $I$. At each $t \in \mathbb{R}$, one can picture the coordinate vector $(d / d v)(t) \in T_{t} \mathbb{R}$ as the unit vector at $t$ in the positive $v$ direction. Then, the velocity vector of $\sigma$ at $t \in I$ is given by

$$
\frac{\partial}{\partial t} \sigma(t)=\sigma_{*}\left(\left.\frac{d}{d v}\right|_{t}\right) \in T_{\sigma(t)} M
$$

where $\sigma_{*}$ denotes the differential of $\sigma$. Intuitively speaking, $\frac{\partial}{\partial t} \sigma(t)$ is the vector rate of change of $\sigma$ at $t \in I$. Let $x_{0}, x_{1}, \ldots, x_{n}$ be a coordinate system in $M$ at a point $\sigma(t)$ of $\sigma$. Then we can express $\frac{\partial}{\partial t} \sigma(t)$ in coordinate form as

$$
\frac{\partial}{\partial t} \sigma(t)=\left.\sum_{a=0}^{n} \frac{d\left(x_{a} \circ \sigma\right)}{d v}(t) \frac{\partial}{\partial x_{a}}\right|_{\sigma(t)} .
$$

Moreover, if $F: M \longrightarrow \bar{M}$ is a map, then $F$ carries $\sigma$ to the curve $(F \circ \sigma)$ : $I \longrightarrow \bar{M}$ in $\bar{M}$. Furthermore, the differential map of $F$ preserves velocities, that is,

$$
F_{*}\left(\frac{\partial}{\partial t} \sigma(t)\right)=\frac{\partial}{\partial t}(F \circ \sigma)(t), \quad \forall t \in I .
$$

A curve $\sigma$ is regular provided $\frac{\partial}{\partial t} \sigma(t) \neq 0$ for all $t$. If $[w, y]$ is a closed interval in $\mathbb{R}$ then a curve segment $\sigma:[w, y] \longrightarrow M$ is a function that has a smooth extension to an open interval containing $[w, y]$. Thus, $\frac{\partial}{\partial t} \sigma(t)$ is well defined even at the endpoints $w$ and $y$. For other properties of the velocity vector $\frac{\partial}{\partial t} \sigma(t)$, see [19].

Next, we adopt the concept of mean curvature flow for Riemannian hypersurfaces given in [13]. For null hypersurfaces, we introduce the null Mean Curvature Flow (MCF) according to the following definition.

Definition 3.2. Let $\left(M^{n+1}, g, S(T M)\right)$ be a compact null hypersurface of a semi-Riemannian manifold $\left(\bar{M}^{n+2}, \bar{g}\right)$. Assume that $F_{0}: M^{n+1} \times[0, T) \longrightarrow$ 
$\bar{M}^{n+2}$ smoothly immerses $M$ as a null hypersurface in $\bar{M}$ such that conditions $\left(C_{1}\right)-\left(C_{7}\right)$ and (3.4)-(3.7) hold. We say that $M_{0}:=F_{0}(M)$ evolves by its null mean curvature vector if there is a whole family $F(\cdot, t)$ of smooth immersions with corresponding hypersurfaces $M_{t}:=F(\cdot, t)(M)$ such that

$$
\frac{\partial F}{\partial t}(p, t)=\mathrm{H}(p, t), \quad F(\cdot, 0)=F_{0}, \quad p \in M^{n+1},
$$

where $\mathrm{H}(p, t):=\mathcal{S}(p, t) N$ is the mean curvature vector of $M_{t}$ at $F(p, t)$.

It is important to notice that in null MCF case, the flow of the surface $M_{t}$ is towards the transversal direction with a pseudo-speed equal to its null mean curvature $\mathcal{S}:=\operatorname{tr}^{s}(B)=\operatorname{tr}^{s}\left(A_{E}^{*}\right)$, as opposed to the flow towards the normal direction in Riemannian (or semi-Riemannian) case. In fact, if $\sigma:[0, T) \longrightarrow M$ is a smooth curve (also, $\sigma$ can be seen as a particle in $M$ ) on an interval $[0, T) \subset \mathbb{R}$, then the action of null isometric immersion $F$ on $M$, that is $F: M \longrightarrow \bar{M}$, gives rise to a new curve (particle) $(F \circ \sigma)$ : $[0, T) \longrightarrow \bar{M}$ in $\bar{M}$. Then, for a given time $t \in[0, T)$ and $p=\sigma(t) \in M$, the velocity vector is given by $(3.14)$ as $\frac{\partial}{\partial t}(F \circ \sigma)(t)$, which is a vector in $\bar{M}$ with a tangential component $\left(\frac{\partial}{\partial t}(F \circ \sigma)(t)\right)^{\top}$ and transversal component $\left(\frac{\partial}{\partial t}(F \circ \sigma)(t)\right)^{t}$. If at every $t \in[0, T)$, we set $\left(\frac{\partial}{\partial t}(F \circ \sigma)(t)\right)^{t}=\mathbf{H}=\mathcal{S} N$, then we obtain the null MCF in the definition above. This choice makes sense due to the fact that tangential velocity has no influence on the shape of the evolving null hypersurface but it controls only the parameterization of the hypersurface (see [17, p. 10] for details).

Example 3.3. In what follows, we show how a double null foliation of Minkowski spacetime evolves under null MCF.

Let $S_{0}$ be an embedded 2-surface and $\Lambda_{0}, \underline{\Lambda}_{0}$ be the null hypersurfaces spanned by outgoing and incoming null geodesics normal to $S_{0}$. Let $\varrho$ : $S_{0} \longrightarrow \mathbb{R}$ be a smooth function (the null lapse, see [5]) on $S_{0}$ and $E^{\prime}$ be a null vector field normal to $S_{0}$ (and tangential to $\Lambda_{0}$ ). Also let $N^{\prime}$ be the null vector field normal to $S_{0}$ and tangent to the null geodesics of $\underline{\Lambda}_{0}$ such that

$$
\bar{g}\left(E^{\prime}, N^{\prime}\right)=-\varrho^{-2} .
$$

Let us extend the fields $E^{\prime}, N^{\prime}$ on $\Lambda_{0}, \underline{\Lambda}_{0}$, respectively, such that $\bar{\nabla}_{E^{\prime}} E^{\prime}=0$ and $\bar{\nabla}_{N^{\prime}} N^{\prime}=0$. Also, extend $\varrho$ to a function on the null hypersurfaces $\Lambda_{0}$ and $\underline{\Lambda}_{0}$ and consider the vector fields $E^{\prime \prime}=\varrho^{2} E^{\prime}$ and $N^{\prime \prime}=\varrho^{2} N^{\prime}$. Define the function $\underline{u}$ on $\Lambda_{0}$ by $E^{\prime \prime} \underline{u}=1$, with $\underline{u}=0$ on $S_{0}$. Similarly, define a function $u$ on $\underline{\Lambda}_{0}$ by $N^{\prime \prime} u=1$, with $u=0$ on $S_{0}$. 
Let $S_{\tilde{\tau}}$ be the embedded 2-surface on $\Lambda_{0}$ such that $\underline{u}=\tilde{\tau}$, and similarly, let $\underline{S}_{\tilde{\tau}}$ be the embedded 2-surface on $\underline{\Lambda}_{0}$ such that $u=\tilde{\tau}$. We also define $N^{\prime}$ on $\Lambda_{0}$ such that $N^{\prime}$ is null and normal to $S_{\tilde{\tau}}$ and $\bar{g}\left(E^{\prime}, N^{\prime}\right)=-\varrho^{-2}$.

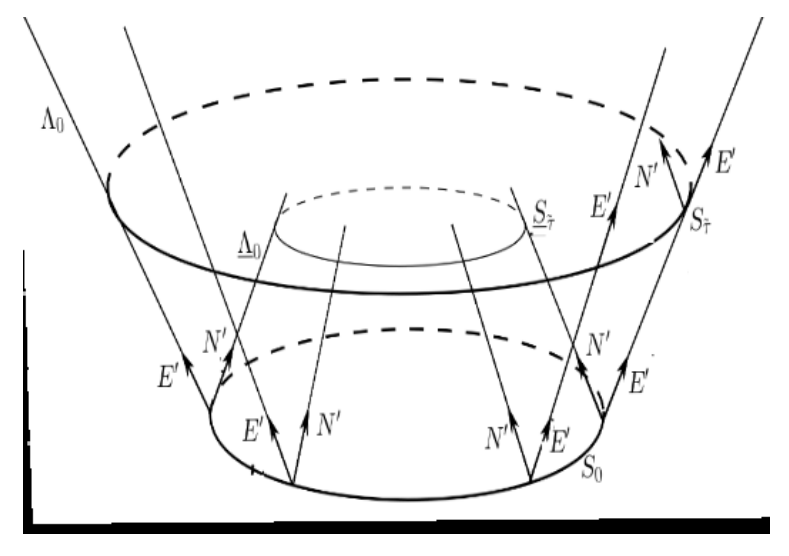

Figure 1. Double conical null foliation.

Consider the affinely parametrized null geodesics which emanate from the points on $S_{\tilde{\tau}}$, with initial tangent vector $N^{\prime}$. These geodesics span null hypersurfaces which we denote by $\underline{C}_{\tilde{\tau}}$. Hence, $\Lambda_{0} \cap \underline{C}_{\tilde{\tau}}=\underline{S}_{\tilde{\tau}}$. Similarly, we define $E^{\prime}$ globally (and the hypersurfaces $C_{\tilde{\tau}}$ such that their normal is $\left.E^{\prime}\right)$. Next, extend $E^{\prime \prime}, N^{\prime \prime}$ to global vector fields such that $E^{\prime \prime}=\varrho^{2} E^{\prime}$ and $N^{\prime \prime}=\varrho^{2} N^{\prime}$. Also, extend the functions $u, \underline{u}$ to global functions such that

$$
E^{\prime \prime} u=0 \quad \text { and } \quad N^{\prime \prime} \underline{u}=0 .
$$

Therefore, $C_{\tilde{\tau}}=\{u=\tilde{\tau}\}$ and $\underline{C}_{\tilde{\tau}}=\{\underline{u}=\tilde{\tau}\}$, and hence $u, \underline{u}$ are optical functions (see [5] for more details). Moreover, the functions $u, \underline{u}$ satisfy the relations

$$
\bar{\nabla} \underline{u}=-N^{\prime}, \quad \bar{\nabla} u=-E^{\prime}, \quad E^{\prime \prime} \underline{u}=1, \quad \text { and } N^{\prime \prime} u=1,
$$

where $\bar{\nabla} u, \bar{\nabla} \underline{u}$ are the gradients of $u, \underline{u}$, respectively. A proof of the above relations can be found in [5]. Observe from the above explanations that we have foliated the spacetime with null hypersurfaces, seen as level-surfaces of $u$ and $\underline{u}$.

Next, we formulate our evolution model from the functions $u$ and $\underline{u}$ as follows. From the previous explanations, we have $\underline{\Lambda}_{0}=\{(x, u(x)): x \in$ $\left.\mathbb{R}^{3}\right\}=\operatorname{Graph}(u) \subset \bar{M}_{1}^{4}$ and $\Lambda_{0}=\left\{(x, \underline{u}(x)): x \in \mathbb{R}^{3}\right\}=\operatorname{Graph}(\underline{u}) \subset \bar{M}_{1}^{4}$. Then, we have the following null immersions $\underline{F}(p, t)=(x(p, t), u(x(p, t), t))$ and $F(p, t)=(x(p, t), \underline{u}(x(p, t), t))$ for $\underline{\Lambda}_{0}, \Lambda_{0}$, respectively. At this point, 
suppose that we want to follow-up the evolution of future cone $\Lambda_{0}$, starting from $S_{0}$, along its transversal direction. Then, observe from Figure 1 that this is essentially a flow along the past cone $\underline{\Lambda}_{0}$; as it is transversal to $\Lambda_{0}$. Also, all points of $\underline{\Lambda}_{0}$ are moved along the transversal direction during this flow. By direct calculations, we have

$$
\frac{\partial \underline{F}}{\partial t}=\left(\frac{\partial x}{\partial t}, \frac{\partial u}{\partial x_{a}} \frac{\partial x_{a}}{\partial t}+\frac{\partial u}{\partial t}\right)=\left(\frac{\partial x}{\partial t}, \bar{g}\left(\bar{\nabla} u, \frac{\partial x}{\partial t}\right)+\frac{\partial u}{\partial t}\right) .
$$

From (3.17) and (3.18) we observe that the vector $\left(N^{\prime \prime}, N^{\prime \prime} \underline{u}\right)=\left(\varrho^{2} N^{\prime}, 0\right)=$ $\left(-\varrho^{2} \bar{\nabla} \underline{u}, 0\right)$ is null and always transversal to the future cone $\Lambda_{0}$, and tangent to the past cone $\underline{\Lambda}_{0}$. Thus, we can write (3.19), using the definition of null $\mathrm{MCF}$, as

$$
\left(\frac{\partial x}{\partial t}, \bar{g}\left(\bar{\nabla} u, \frac{\partial x}{\partial t}\right)+\frac{\partial u}{\partial t}\right)=\mathcal{S}\left(-\varrho^{2} \bar{\nabla} \underline{u}, 0\right) .
$$

Then from (3.20), we get

$$
\frac{\partial x}{\partial t}=-\mathcal{S} \varrho^{2} \bar{\nabla} \underline{u} \text { and } \bar{g}\left(\bar{\nabla} u, \frac{\partial x}{\partial t}\right)+\frac{\partial u}{\partial t}=0 .
$$

From (3.21), (3.16) and (3.18) we deduce that

$$
\frac{\partial u}{\partial t}=\mathcal{S} \varrho^{2} \bar{g}(\bar{\nabla} u, \bar{\nabla} \underline{u})=-\mathcal{S} \varrho^{2} \varrho^{-2}=-\mathcal{S} .
$$

Finally, using (2.4), (3.21) and (3.22), we have $\frac{\partial x}{\partial t}=-\varrho^{2} \operatorname{div}(\bar{\nabla} u) \bar{\nabla} \underline{u}$ and $\frac{\partial u}{\partial t}=-\operatorname{div}(\bar{\nabla} u)$. These PDE's describes the flow of the above cones under null MCF.

\section{Short-time existence and evolution of geometric quantities}

For a Lorentzian ambient manifold, the null MCF equation (3.15) represents a strictly non-linear PDE. To show this, let $\left(\mathcal{U} ; x_{0}, \ldots, x_{n}\right)$ be local co-ordinates around $p \in \mathcal{U} \subset M^{n+1}$ at $t \in[0, T)$, such that the local vector fields $X_{0}=E=\frac{\partial}{\partial x_{0}}, X_{1}=\frac{\partial}{\partial x_{1}}, \ldots, X_{n}=\frac{\partial}{\partial x_{n}}$ and $N=\frac{\partial}{\partial x_{n+1}}$ spans $T \bar{M}$ at time instant $t$. Let us write $F:=F(p, t), \mathrm{H}:=\mathrm{H}(p, t)$ and $E_{a}:=F_{*} X_{a}$, for any $a \in\{0, \ldots, n\}$. As $F$ is a null isometric immersion, [7, p. 109] shows that $F$ is an affine immersion, that is, for any $a, b \in\{0, \ldots, n\}$ we have $\bar{\nabla}_{E_{a}} E_{b}=F_{*}\left(\nabla_{X_{a}} X_{b}\right)+B\left(X_{a}, X_{b}\right) N$ and thus, since $B\left(X_{0}, X_{0}\right)=B(E, E)=0$, then the null mean curvature vector $\mathrm{H}$ can 
be written, for all $\alpha, \beta, \gamma, \mu \in\{1, \ldots, n\}$, as

$$
\begin{aligned}
\mathrm{H} & =\left(g^{\alpha \beta} \bar{\nabla}_{E_{\alpha}} E_{\beta}\right)^{t}=g^{\alpha \beta} \bar{\nabla}_{E_{\alpha}} E_{\beta}-\left(g^{\alpha \beta} \bar{\nabla}_{E_{\alpha}} E_{\beta}\right)^{\top} \\
& =g^{\alpha \beta} \bar{\nabla}_{E_{\alpha}} E_{\beta}-\bar{g}\left(g^{\alpha \beta} \bar{\nabla}_{E_{\alpha}} E_{\beta}, E_{\gamma}\right) g^{\gamma \mu} E_{\mu},
\end{aligned}
$$

where $(\cdot)^{\top}$ and $(\cdot)^{t}$ are the tangential and transversal components of $(\cdot)$ respectively. Let $\bar{\Gamma}_{\alpha \beta}^{a}$ denote the $a$-th component of $\bar{\nabla}_{E_{\alpha}} E_{\beta}$ in the basis $\left\{E_{0}, \ldots, E_{n}\right\}$, then (4.1) and (3.15) gives $\frac{\partial F}{\partial t}=g^{\alpha \beta} \bar{\Gamma}_{\alpha \beta}^{a}\left(E_{a}-g^{\gamma \mu} g_{a \gamma} E_{\mu}\right)$, which is a system of non-linear PDE's because $\left(g^{\alpha \beta}\right)$, for $\alpha, \beta \in\{1, \ldots, n\}$, is a positive definite matrix which only depends on the first derivatives of $F$. Moreover, using the local theory of parabolic PDE's, the geometric equation in (3.15) can be shown to be equivalent to a quasilinear scalar equation, which may be solved for some short interval of time using linearization techniques. This short-time solution may then be continued as long as it does not become singular [22].

Suppose that the null hypersurface $(M, g)$ moves by null mean curvature and let $F$ be the smooth immersion in Definition 3.2. Notice that the null immersion $F$ has no well-defined D' Alambertian operator, but we can compute its D' Alambertian-type operator $\Delta^{g} F$ via the D' Alambertians of its coordinate functions with respect to $\widehat{g}$ (see [4]). Alternatively, we can compute $\Delta^{g} F$ from the Hessian $\operatorname{Hess}^{g}(F)$ (regarded as a vector-valued function on $\left.M_{0}=F(M)\right)$ of $F$. Let $(M, g)$ be a null hypersurface of $(\bar{M}, \bar{g})$, then

$$
\Delta^{g} F=\operatorname{tr}\left(\operatorname{Hess}^{g}(F)\right),
$$

where $\operatorname{Hess}^{g}(F)=X\left(F_{*} Y\right)-F_{*}\left(\nabla_{X}^{\prime} Y\right)$, for all $X, Y \in \Gamma(T M)$ and trace is taken on $M_{0}$ with respect to $\widehat{g}$. In fact, using (3.5) we have

$$
\begin{aligned}
\operatorname{Hess}^{g}(F) & =X_{a}\left(F_{*} X_{b}\right)-F_{*}\left(\nabla_{X_{a}}^{\prime} X_{b}\right) \\
& =X_{a}\left(F_{*} X_{b}\right)-\left(\nabla_{F_{*} X_{a}} F_{*} X_{b}\right), \quad \forall, a, b \in\{0, \ldots, n\} .
\end{aligned}
$$

Contracting (4.3) with respect to $\widehat{g}$, we get

$$
\begin{aligned}
\operatorname{tr}\left(\operatorname{Hess}^{g}(F)\right) & =g^{[a b]}\left(X_{a}\left(F_{*} X_{b}\right)-\left(\nabla_{F_{*} X_{a}} F_{*} X_{b}\right)\right) \\
& =g^{[a b]} B\left(F_{*} X_{a}, F_{*} X_{b}\right) N .
\end{aligned}
$$

As $F$ is also an affine immersion [7, p. 109], (4.4) vanishes when $a=0$ or $b=0$ due to the fact that the local second fundamental form $B$ of a null hypersurface is degenerate. Thus, from (4.4) and (4.1), we deduce that

$$
\Delta^{g} F=\operatorname{tr}\left(\operatorname{Hess}^{g}(F)\right)=\mathrm{H} .
$$


Now, from (4.5) we state the following.

Proposition 4.1. Let $(M, g)$ be a null hypersurface in a Lorentzian manifold $(\bar{M}, \bar{g})$ and $F_{0}: M^{n+1} \longrightarrow \bar{M}^{n+2}$ a given immersion. Then, the null-MCF equation in Definition 3.2 is given by

$$
\frac{\partial F}{\partial t}=\Delta^{g} F
$$

Using Proposition 4.1 we can state and prove an existence theorem for the null-MCF in Definition 3.2 for a short time interval, for a compact null hypersurface.

Theorem 4.2 (Short-time existance). Let $(M, g)$ a compact null hypersurface of a Lorentzian manifold $(\bar{M}, \bar{g})$ and $F_{0}: M^{n+1} \longrightarrow \bar{M}^{n+2}$ a given immersion. There exists a positive constant $T>0$ and a unique smooth family of immersions $F(\cdot, t): M^{n+1} \longrightarrow \bar{M}^{n+1}$ such that

$$
\frac{\partial F}{\partial t}(p, t)=\mathrm{H}(p, t), \quad F(\cdot, 0)=F_{0},
$$

where $p \in M$ and $t \in[0, T)$.

Proof. We start off by observing that $F(\cdot, 0)=F_{0}$ is an immersion, so $F(p, t)$ would be an immersion for some small $t$ (see, for instance [12, p. $35])$. Hence, we only focus on the existence and solution of the PDE above. Let us consider a vector field $U=u^{a} \frac{\partial}{\partial x_{a}}$ such that $\frac{\partial \tilde{F}}{\partial t}=\Delta^{g} \tilde{F}+u^{a} \frac{\partial \tilde{F}}{\partial x_{a}}$ has a solution for initial data $F_{0}, \tilde{F}: M \times[0, T) \longrightarrow \bar{M}^{n+1}$. We establish that the same happens to the null mean curvature flow with initial data $F_{0}$. Consider a family $\varphi_{t}: M \longrightarrow M$ of diffeomorphisms of $M$. Set $F(p):=\tilde{F}\left(\varphi_{t}(p), t\right)$, where $\tilde{F}$ is as mentioned above. Then at a point $p \in M$, we have

$$
\begin{aligned}
\frac{\partial F}{\partial t}(p) & =\frac{\partial \tilde{F}}{\partial x_{a}}\left(\varphi_{t}(p), t\right) \frac{\partial \varphi_{t}^{a}}{\partial t}(p)+\frac{\partial \tilde{F}}{\partial t}\left(\varphi_{t}(p), t\right) \\
& =\Delta^{g} \tilde{F}\left(\varphi_{t}(p), t\right)+\frac{\partial \tilde{F}}{\partial x_{a}}\left(\varphi_{t}(p), t\right)\left(u^{a}+\frac{\partial \varphi_{t}^{a}}{\partial t}(p)\right) .
\end{aligned}
$$

Therefore, by (4.8), to get a solution to the null-MCF equation is enough to find a family $\varphi_{t}$ such that:

$$
\frac{\partial \varphi_{t}}{\partial t}=-U, \quad \varphi_{0}=\mathrm{id}
$$

Equation (4.9) is an initial value problem for a system of ODE's, so we can find a solution to it. Moreover, taking $T>0$ small enough we can assume that $\varphi_{t}$ is a diffeomorphism, for any $t \in[0, T]$, reason being; the initial data is a diffeomorphism (the identity) and the fact that the diffeomorphisms 
from a compact manifold into itself form a stable class (see [12, p. 35]). Therefore, $F(p):=\tilde{F}\left(\varphi_{t}(p), t\right)$ represents a solution of null-MCF equation with initial data $F_{0}$. In fact; $\frac{\partial F}{\partial t}(p)=\Delta^{g} \tilde{F}\left(\varphi_{t}(p), t\right)=\Delta^{g} F(p)$ and $F(p, 0)=$ $\tilde{F}\left(\varphi_{0}(p), 0\right)=\tilde{F}(\operatorname{id}(p), 0)=F_{0}(p)$. Finally, we show that $\frac{\partial \tilde{F}}{\partial t}=\Delta^{g} \tilde{F}+u^{a} \frac{\partial \tilde{F}}{\partial x_{a}}$ has a solution. In this regard, let us consider the vector $U$ whose coordinates are $u^{a}=g^{[b c]}\left(\Gamma_{b c}^{a}-\left(\Gamma_{0}\right)_{b c}^{a}\right)$, where $\Gamma_{b c}^{a}$ are the coefficients of the induced connection on $M$, in which $\left(\Gamma_{0}\right)_{b c}^{a}$ denotes the coefficient at $t=0$. Then by (4.4), we have

$$
\frac{\partial \tilde{F}}{\partial t}=\Delta^{g} \tilde{F}+u^{a} \frac{\partial \tilde{F}}{\partial x_{a}}=g^{[b c]}\left(\frac{\partial^{2} \tilde{F}}{\partial x_{b} \partial x_{c}}-\left(\Gamma_{0}\right)_{b c}^{a} \frac{\partial \tilde{F}}{\partial x_{a}}\right),
$$

which is a system of quasilinear parabolic PDE's because $\left(g^{[b c]}\right)$ is a positive definite matrix which only depends on the first derivatives of $\tilde{F}$. Hence, the local theory of parabolic PDE's (see, for instance, [22]) and the fact that $M$ is compact, gives us the existence and uniqueness of the solution in a short interval of time $[0, T)$, which completes the proof.

Now, we discuss the extrinsic geometry of $M_{t}$. To that end let us set $E_{a}:=$ $F_{*} X_{a}=\frac{\partial F}{\partial x_{a}}, \tau_{a}:=\tau\left(E_{a}\right), B_{\alpha \beta}=B\left(E_{\alpha}, E_{\beta}\right), C_{\alpha \beta}=C\left(E_{\alpha}, E_{\beta}\right)$ and $\tilde{\mathcal{S}}:=$ $\sum_{\alpha=1}^{n} C\left(E_{\alpha}, E_{\alpha}\right)$.

Theorem 4.3. Let $(M, g)$ be a screen integrable null hypersurface of a Lorentzian manifold $(\bar{M}, \bar{g})$. Let $F_{0}: M \longrightarrow \bar{M}$ be a given immersion. On any solution $M_{t}$ of null MCF (3.15), the induced objects $g, B, C, \mathcal{S}$, and the null normal $E$ evolve as follows

$$
\begin{gathered}
\frac{\partial}{\partial t} g_{\alpha \beta}=-2 \mathcal{S} C_{\alpha \beta}, \\
\frac{\partial}{\partial t} g^{\alpha \beta}=2 \mathcal{S} C^{\alpha \beta}, \\
\frac{\partial}{\partial t} E=-\nabla^{s} \mathcal{S}-\mathcal{S} g^{\alpha \beta} \tau_{\alpha} E_{\beta}, \\
\frac{\partial}{\partial t} B_{\alpha \beta}=\operatorname{Hess}^{s}(\mathcal{S})-\mathcal{S} B\left(\frac{\partial}{\partial x_{\alpha}}, A_{N} \frac{\partial}{\partial x_{\beta}}\right)+\frac{\partial}{\partial x_{\beta}}(\mathcal{S}) \tau_{\alpha} \\
+\frac{\partial}{\partial x_{\alpha}}(\mathcal{S}) \tau_{\beta}+\mathcal{S} \frac{\partial}{\partial x_{\alpha}}\left(\tau_{\beta}\right)+\mathcal{S} \tau_{\alpha} \tau_{\beta}-\mathcal{S} \Gamma_{\alpha \beta}^{* \gamma} \tau_{\gamma},
\end{gathered}
$$




$$
\begin{aligned}
\frac{\partial}{\partial t} \mathcal{S} & =\Delta \mathcal{S}+\mathcal{S H}+g^{\alpha \beta}\left(\frac{\partial}{\partial x_{\beta}}(\mathcal{S}) \tau_{\alpha}\right. \\
& \left.\left.+\frac{\partial}{\partial x_{\alpha}}(\mathcal{S}) \tau_{\beta}+\mathcal{S} \frac{\partial}{\partial x_{\alpha}}\left(\tau_{\beta}\right)+\mathcal{S} \tau_{\alpha} \tau_{\beta}-\mathcal{S} \Gamma_{\alpha \beta}^{* \gamma} \tau_{\gamma}\right)\right),
\end{aligned}
$$

where $\mathcal{H}:=C^{\alpha \beta} B_{\alpha \beta}=\operatorname{tr}^{s}\left(A_{E}^{*} \circ A_{N}\right)$, for any $\alpha, \beta, \gamma \in\{1, \ldots, n\}$.

Proof. By virtue of (3.4), the null MCF equation (3.15) and the local symmetry of the Levi-Civita connection, we derive

$$
\begin{aligned}
\frac{\partial}{\partial t} g_{\alpha \beta} & =\frac{\partial}{\partial t} \bar{g}\left(E_{\alpha}, E_{\beta}\right)=\bar{g}\left(\frac{\partial}{\partial t} E_{\alpha}, E_{\beta}\right)+\bar{g}\left(E_{\alpha}, \frac{\partial}{\partial t} E_{\beta}\right) \\
& =\bar{g}\left(\frac{\partial}{\partial x_{\alpha}} \mathrm{H}, E_{\beta}\right)+\bar{g}\left(E_{\alpha}, \frac{\partial}{\partial x_{\beta}} \mathrm{H}\right) .
\end{aligned}
$$

As $F$ preserves both the radical and screen subspaces (see [7, p.104]), we can see, by (3.7), that $E_{\alpha}$ belongs to the screen distribution in $M_{t}$, for all $\alpha \in\{1, \ldots, n\}$. From the above facts and that the mean curvature vector $\mathbf{H}$ is transversal to $M_{t}$, we get $\bar{g}\left(E_{\alpha}, \mathrm{H}\right)=0$. Hence, from the Gauss-Codazzi equations (2.1), (2.3), we get

$$
\bar{g}\left(\frac{\partial}{\partial x_{\alpha}} \mathrm{H}, E_{\beta}\right)=-\bar{g}\left(\mathrm{H}, \frac{\partial}{\partial x_{\alpha}} E_{\beta}\right)=-\mathcal{S} C_{\alpha \beta} .
$$

Then (4.11) follows from (4.16) and (4.17) and the symmetry of $C$ in the indices $\alpha$ and $\beta$. Relation (4.12) follows easily from (4.11) by product rule. Next, we prove (4.13). Decomposing the vector field $\frac{\partial}{\partial t} E$ in the basis $\left\{E_{1}, \ldots, E_{n}\right\}$ and observing $\bar{g}\left(E, E_{a}\right)=0$, for all $a \in\{0, \ldots, n\}$, we have

$$
\begin{aligned}
\frac{\partial}{\partial t} E & =g^{\alpha \beta} \bar{g}\left(\frac{\partial}{\partial t} E, E_{\alpha}\right) E_{\beta}=g^{\alpha \beta}\left(\frac{\partial}{\partial t} \bar{g}\left(E, E_{\alpha}\right)-\bar{g}\left(E, \frac{\partial}{\partial t} E_{\alpha}\right)\right) E_{\beta} \\
& =-g^{\alpha \beta}\left(\frac{\partial}{\partial x_{\alpha}} \bar{g}(E, \mathrm{H})-\bar{g}\left(\frac{\partial}{\partial x_{\alpha}} E, \mathrm{H}\right)\right) E_{\beta},
\end{aligned}
$$

in which we have used the symmetry of the Levi-Civita connection. But $\bar{g}(E, \mathrm{H})=\bar{g}(E, \mathcal{S} N)=\mathcal{S} \bar{g}(E, N)=\mathcal{S}$ and by (2.1) and (2.4) the second term in the parenthesis of (4.18) simplifies as

$$
\bar{g}\left(\frac{\partial}{\partial x_{\alpha}} E, \mathrm{H}\right)=\mathcal{S} \bar{g}\left(\frac{\partial}{\partial x_{\alpha}} E, N\right)=-\mathcal{S} \tau_{\alpha} .
$$

Placing (4.19) back into (4.18), we get

$$
\frac{\partial}{\partial t} E=-g^{\alpha \beta} \frac{\partial}{\partial x_{\alpha}}(\mathcal{S}) E_{\beta}=-\nabla^{s} \mathcal{S}-\mathcal{S} g^{\alpha \beta} \tau_{\alpha} E_{\beta}
$$


which proves (4.13). From the Gauss formula (2.1) we derive

$$
\begin{aligned}
\frac{\partial}{\partial t} B_{\alpha \beta} & =\frac{\partial}{\partial t} \bar{g}\left(\frac{\partial}{\partial x_{\alpha}} E_{\beta}, E\right) \\
& =\bar{g}\left(\frac{\partial^{2}}{\partial t \partial x_{\alpha}} E_{\beta}, E\right)+\bar{g}\left(\frac{\partial}{\partial x_{\alpha}} E_{\beta}, \frac{\partial}{\partial t} E\right)
\end{aligned}
$$

As we are working in local coordinates and that $\bar{R}=0$ (follows easily from Gauss-Codazzi equations and $\left(C_{7}\right)$, see details in $\left.[7,103]\right)$, one gets $\frac{\partial^{2}}{\partial t \partial x_{\alpha}} E_{\beta}=\frac{\partial^{2}}{\partial x_{\alpha} \partial t} E_{\beta}=\frac{\partial^{2}}{\partial x_{\alpha} \partial x_{\beta}} \mathbf{H}$ and so (4.20) gives

$$
\frac{\partial}{\partial t} B_{\alpha \beta}=\bar{g}\left(\frac{\partial^{2}}{\partial x_{\alpha} \partial x_{\beta}} \mathbf{H}, E\right)+\bar{g}\left(\frac{\partial}{\partial x_{\alpha}} E_{\beta}, \frac{\partial}{\partial t} E\right) .
$$

Now, using Gauss-Codazzi relations (2.1)-(2.2) together with $\frac{\partial}{\partial x_{\beta}} \mathrm{H}=\frac{\partial}{\partial x_{\beta}}(\mathcal{S} N)=$ $\left(\frac{\partial}{\partial x_{\beta}} \mathcal{S}\right) N+\mathcal{S}\left(\frac{\partial}{\partial x_{\beta}} N\right)$, gives

$$
\begin{aligned}
\frac{\partial}{\partial t} B_{\alpha \beta} & =\bar{g}\left(\frac{\partial}{\partial x_{\alpha}}\left(\left(\frac{\partial}{\partial x_{\beta}}(\mathcal{S})\right) N\right), E\right)-\mathcal{S} B\left(\frac{\partial}{\partial x_{\alpha}}, A_{N} \frac{\partial}{\partial x_{\beta}}\right) \\
& +\frac{\partial}{\partial x_{\alpha}}(\mathcal{S}) \tau_{\beta}+\mathcal{S} \frac{\partial}{\partial x_{\alpha}}\left(\tau_{\beta}\right)+\mathcal{S} \tau_{\alpha} \tau_{\beta}+\bar{g}\left(\frac{\partial}{\partial x_{\alpha}} E_{\beta}, \frac{\partial}{\partial t} E\right) \\
& =\frac{\partial}{\partial x_{\alpha}}\left(\frac{\partial}{\partial x_{\beta}}(\mathcal{S})\right)-\mathcal{S} B\left(\frac{\partial}{\partial x_{\alpha}}, A_{N} \frac{\partial}{\partial x_{\beta}}\right)+\frac{\partial}{\partial x_{\beta}}(\mathcal{S}) \tau_{\alpha} \\
& +\frac{\partial}{\partial x_{\alpha}}(\mathcal{S}) \tau_{\beta}+\mathcal{S} \frac{\partial}{\partial x_{\alpha}}\left(\tau_{\beta}\right)+\mathcal{S} \tau_{\alpha} \tau_{\beta}+\bar{g}\left(\frac{\partial}{\partial x_{\alpha}} E_{\beta}, \frac{\partial}{\partial t} E\right) .
\end{aligned}
$$

Then applying (4.13) to (4.22) we get

$$
\begin{aligned}
\frac{\partial}{\partial t} B_{\alpha \beta} & =\frac{\partial}{\partial x_{\alpha}}\left(\frac{\partial}{\partial x_{\beta}}(\mathcal{S})\right)-\mathcal{S} B\left(\frac{\partial}{\partial x_{\alpha}}, A_{N} \frac{\partial}{\partial x_{\beta}}\right)+\frac{\partial}{\partial x_{\beta}}(\mathcal{S}) \tau_{\alpha} \\
& +\frac{\partial}{\partial x_{\alpha}}(\mathcal{S}) \tau_{\beta}+\mathcal{S} \frac{\partial}{\partial x_{\alpha}}\left(\tau_{\beta}\right)+\mathcal{S} \tau_{\alpha} \tau_{\beta}-g^{\gamma \mu} \bar{g}\left(\frac{\partial}{\partial x_{\alpha}} E_{\beta}, \frac{\partial}{\partial x_{\gamma}}(\mathcal{S}) E_{\mu}\right) \\
& -\mathcal{S} g^{\gamma \mu} \tau_{\gamma} \bar{g}\left(\frac{\partial}{\partial x_{\alpha}} E_{\beta}, E_{\mu}\right) \\
& =\frac{\partial}{\partial x_{\alpha}}\left(\frac{\partial}{\partial x_{\beta}}(\mathcal{S})\right)-\mathcal{S} B\left(\frac{\partial}{\partial x_{\alpha}}, A_{N} \frac{\partial}{\partial x_{\beta}}\right)+\frac{\partial}{\partial x_{\beta}}(\mathcal{S}) \tau_{\alpha} \\
& \left.+\frac{\partial}{\partial x_{\alpha}}(\mathcal{S}) \tau_{\beta}+\mathcal{S} \frac{\partial}{\partial x_{\alpha}}\left(\tau_{\beta}\right)+\mathcal{S} \tau_{\alpha} \tau_{\beta}-\Gamma_{\alpha \beta}^{* \gamma} \frac{\partial}{\partial x_{\gamma}}(\mathcal{S})-\mathcal{S} \Gamma_{\alpha \beta}^{* \gamma} \tau_{\gamma}, \quad, 4.23\right)
\end{aligned}
$$


where $\Gamma_{\alpha \beta}^{* \gamma}$ are the coefficient of the metric connection $\nabla^{*}$. Then, from (4.23) we deduce that

$$
\begin{aligned}
\frac{\partial}{\partial t} B_{\alpha \beta} & =\operatorname{Hess}^{S}(\mathcal{S})-\mathcal{S} B\left(\frac{\partial}{\partial x_{\alpha}}, A_{N} \frac{\partial}{\partial x_{\beta}}\right)+\frac{\partial}{\partial x_{\beta}}(\mathcal{S}) \tau_{\alpha} \\
& +\frac{\partial}{\partial x_{\alpha}}(\mathcal{S}) \tau_{\beta}+\mathcal{S} \frac{\partial}{\partial x_{\alpha}}\left(\tau_{\beta}\right)+\mathcal{S} \tau_{\alpha} \tau_{\beta}-\mathcal{S} \Gamma_{\alpha \beta}^{* \gamma} \tau_{\gamma}
\end{aligned}
$$

where $\operatorname{Hess}^{s}(\mathcal{S}):=\frac{\partial}{\partial x_{\alpha}}\left(\frac{\partial}{\partial x_{\beta}}(\mathcal{S})\right)-\Gamma_{* \alpha \beta}^{\gamma} \frac{\partial}{\partial x_{\gamma}}(\mathcal{S})$, which proves (4.14). Finally, we have from (4.12) and (4.14) that

$$
\begin{aligned}
\frac{\partial}{\partial t} \mathcal{S} & =\left(\frac{\partial}{\partial t} g^{\alpha \beta}\right) B_{\alpha \beta}+g^{\alpha \beta}\left(\frac{\partial}{\partial t} B_{\alpha \beta}\right) \\
& =2 \mathcal{S} C^{\alpha \beta} B_{\alpha \beta}+g^{\alpha \beta}\left(\operatorname{Hess}^{s}(\mathcal{S})-\mathcal{S} g\left(A_{N} E_{\beta}, A_{E}^{*} E_{\alpha}\right)+\frac{\partial}{\partial x_{\beta}}(\mathcal{S}) \tau_{\alpha}\right. \\
& \left.+\frac{\partial}{\partial x_{\alpha}}(\mathcal{S}) \tau_{\beta}+\mathcal{S} \frac{\partial}{\partial x_{\alpha}}\left(\tau_{\beta}\right)+\mathcal{S} \tau_{\alpha} \tau_{\beta}-\mathcal{S} \Gamma_{\alpha \beta}^{* \gamma} \tau_{\gamma}\right) \\
& =\Delta^{s} \mathcal{S}+2 \mathcal{S} C^{\alpha \beta} B_{\alpha \beta}-\mathcal{S} \operatorname{tr}\left(A_{E}^{*} \circ A_{N}\right) \\
& \left.+g^{\alpha \beta}\left(\frac{\partial}{\partial x_{\beta}}(\mathcal{S}) \tau_{\alpha}+\frac{\partial}{\partial x_{\alpha}}(\mathcal{S}) \tau_{\beta}+\mathcal{S} \frac{\partial}{\partial x_{\alpha}}\left(\tau_{\beta}\right)+\mathcal{S} \tau_{\alpha} \tau_{\beta}-\mathcal{S} \Gamma_{\alpha \beta}^{* \gamma} \tau_{\gamma}\right)\right),
\end{aligned}
$$

which proves (4.15) and the proof ends here.

Next, we will derive the evolution equation for the squared norm (with respect to the screen distribution) $\left|A_{E}^{*}\right|_{s}^{2}$ of screen shape operator $A_{E}^{*}$. To that end, let us consider an orthonormal basis $\left\{X_{1}, \ldots, X_{n}\right\}$, where $X_{\alpha}=$ $\frac{\partial}{\partial x_{\alpha}}$, of $S(T M)$ around a point $p \in M$ such that the induced Levi-Civita connection $\nabla^{*}$ of $S(T M)$ satisfy $\left(\nabla_{X_{\alpha}}^{*} X_{\beta}\right)(p)=0$ and $C\left(X_{0}, X_{\alpha}\right)=0$. Observe that the above assumptions implies that, locally the curvature $R^{*}$ of $S(T M)$ vanishes. Furthermore, we will assume that the 1 -form $\tau$ vanishes on $S(T M)$. As an example we have the following.

Example 4.4 (Null cone of $\mathbb{R}_{1}^{n+2}$ ). Let $\mathbb{R}_{1}^{n+2}$ be the space $\mathbb{R}^{n+2}$ endowed with a semi-Euclidean metric

$$
\bar{g}(x, y)=-x_{0} y_{0}+\sum_{a=0}^{n+1} x_{a} y_{a}, \quad\left(x=\sum_{A=0}^{n+1} x^{A} \partial x_{A}\right),
$$

where $\partial x_{A}:=\frac{\partial}{\partial x_{A}}$. Then, the null cone $\Lambda_{0}^{n+1}$ is given by the equation $x_{0}^{2}=\sum_{a=1}^{n+1} x_{a}^{2}, x_{0} \neq 0$. It is well-known (for example see the books $[7,8]$ ) that $\Lambda_{0}^{n+1}$ is a null hypersurface of $\mathbb{R}_{1}^{n+2}$, in which the radical distribution is spanned by a global vector field $E=\sum_{A=0}^{n+1} x_{A} \partial x_{A}$ on $\Lambda_{0}^{n+1}$. The transversal bundle is spanned by a global section $N$ given by $N=\frac{1}{2 x_{0}^{2}}\left\{-x_{0} \partial x_{0}+\right.$ 
$\left.\sum_{a=1}^{n+1} x_{a} \partial x_{a}\right\}$. Moreover, $E$ being the position vector field, one gets $\bar{\nabla}_{X} E=$ $\nabla_{X} E=X$, for any $X \in \Gamma(T M)$. Consequently, $A_{E}^{*} X+\tau(X) E+X=0$. Noticing that the operator $A_{E}^{*}$ is screen-valved, we infer from the last relation that

$$
A_{E}^{*} X=-P X, \quad \tau(X)=-\bar{g}(X, N)=-\lambda(X),
$$

for any $X \in \Gamma(T M)$. Next, any $X \in \Gamma\left(S\left(T \Lambda_{0}^{n+1}\right)\right)$ is expressed as $X=$ $\sum_{a=1}^{n+1} \tilde{X}_{a} \partial x_{a}$, where $\left\{\tilde{X}_{1}, \ldots, \tilde{X}_{n+1}\right\}$ satisfy $\sum_{a=1}^{n+1} x_{a} \tilde{X}_{a}=0$. From the second relation of (4.24) we can clearly see that $\tau(X)=0$ for any $X \in$ $\Gamma(S(T M))$.

Next, we turn our attention to the evolution equation of $\left|A_{E}^{*}\right|_{s}^{2}$. To that end, we will need the following result.

Proposition 4.5. Let $(M, g)$ be a screen integrable null hypersurface of a Lorentzian manifold $(\bar{M}, \bar{g})$. The local second fundamental form $B$ satisfy

$$
\Delta^{s} B_{\alpha \beta}=\operatorname{Hess}^{s}(\mathcal{S})-\mathcal{H} B_{\alpha \beta}+\mathcal{S} C\left(X_{\alpha}, A_{E}^{*} X_{\beta}\right),
$$

for all $\alpha, \beta \in\{1, \ldots, n\}$, where $\mathcal{H}:=\operatorname{tr}^{s}\left(A_{E}^{*} \circ A_{N}\right)$.

Proof. By the assumption $\bar{R}=0$, the Gauss-Codazzi equation (3.1) of null hypersurfaces given in $[7$, p. 93] implies

$$
\left(\nabla_{X} h\right)(Y, Z)=\left(\nabla_{Y} h\right)(X, Z),
$$

for all $X, Y, Z \in \Gamma(T M)$, where

$$
\left(\nabla_{X} h\right)(Y, Z)=\nabla_{X}^{t} h(Y, Z)-h\left(\nabla_{X} Y, Z\right)-h\left(Y, \nabla_{X} Z\right) .
$$

As $\nabla_{X_{\alpha}}^{*} X_{\beta}=0$, we have

$$
h\left(\nabla_{X_{\alpha}} X_{\beta}, X_{\gamma}\right)=h\left(\nabla_{X_{\alpha}}^{*} X_{\beta}, X_{\gamma}\right)+C\left(X_{\alpha}, X_{\beta}\right) h\left(E, X_{\gamma}\right)=0,
$$

in which we have considered (2.3) and the fact $h(E, X)=0$, for any $X \in$ $\Gamma(T M)$. Thus, (4.25) and (4.26) implies

$$
\nabla_{X_{\gamma}}^{t} h\left(X_{\alpha}, X_{\beta}\right)=\nabla_{X_{\alpha}}^{t} h\left(X_{\gamma}, X_{\beta}\right)
$$

Differentiating (4.27) and applying the definition of curvature, we get

$$
\begin{array}{rl}
\nabla_{X_{\mu}}^{t} \nabla_{X_{\gamma}}^{t} & h\left(X_{\alpha}, X_{\beta}\right)=\nabla_{X_{\mu}}^{t} \nabla_{X_{\alpha}}^{t} h\left(X_{\gamma}, X_{\beta}\right) \\
= & \nabla_{X_{\alpha}}^{t} \nabla_{X_{\mu}}^{t} h\left(X_{\gamma}, X_{\beta}\right)-\left(R\left(X_{\alpha}, X_{\mu}\right) h\right)\left(X_{\beta}, X_{\gamma}\right)
\end{array}
$$


where

$$
\begin{aligned}
\left(R\left(X_{\alpha}, X_{\mu}\right) h\right) & \left(X_{\beta}, X_{\gamma}\right)=R^{t}\left(X_{\alpha}, X_{\mu}\right) h\left(X_{\beta}, X_{\gamma}\right) \\
& -h\left(R\left(X_{\alpha}, X_{\mu}\right) X_{\beta}, X_{\gamma}\right)-h\left(X_{\beta}, R\left(X_{\alpha}, X_{\mu}\right) X_{\gamma}\right),
\end{aligned}
$$

and $R^{t}$ is the curvature tensor of the transversal bundle, given by

$$
R^{t}(X, Y) N=\nabla_{X}^{t} \nabla_{Y}^{t} N-\nabla_{Y}^{t} \nabla_{X}^{t} N-\nabla_{[X, Y]}^{t} N
$$

for any $X, Y \in \Gamma(T M)$ and $N \in \Gamma(\operatorname{tr}(T M))$. Then applying (4.28), (4.29) and (4.30) while considering $\nabla_{X}^{t} N=\tau(X) N$, we get

$$
\begin{aligned}
\nabla_{X_{\mu}}^{t} \nabla_{X_{\gamma}}^{t} h\left(X_{\alpha}, X_{\beta}\right) & =\nabla_{X_{\alpha}}^{t} \nabla_{X_{\mu}}^{t} h\left(X_{\beta}, X_{\gamma}\right)+h\left(R\left(X_{\alpha}, X_{\mu}\right) X_{\beta}, X_{\gamma}\right) \\
& +h\left(X_{\beta}, R\left(X_{\alpha}, X_{\mu}\right) X_{\gamma}\right) \\
& =\nabla_{X_{\alpha}}^{t} \nabla_{X_{\beta}}^{t} h\left(X_{\mu}, X_{\gamma}\right)+h\left(R\left(X_{\alpha}, X_{\mu}\right) X_{\beta}, X_{\gamma}\right) \\
& +h\left(X_{\beta}, R\left(X_{\alpha}, X_{\mu}\right) X_{\gamma}\right),
\end{aligned}
$$

where in the last equality we have used (4.27). Now, using the fact $h(X, Y)=$ $B(X, Y) N, B(X, Y)=g\left(A_{E}^{*} X, Y\right)$, for any $X, Y \in \Gamma(T M)$, and the assumption $\tau=0$ on the screen distribution, (4.31) reduces to

$$
\begin{aligned}
X_{\mu}\left(X_{\gamma}\left(B_{\alpha \beta}\right)\right) & =X_{\alpha}\left(X_{\beta}\left(B_{\mu \gamma}\right)\right)+B\left(R\left(X_{\alpha}, X_{\mu}\right) X_{\beta}, X_{\gamma}\right) \\
& +B\left(X_{\beta}, R\left(X_{\alpha}, X_{\mu}\right) X_{\gamma}\right) \\
& =X_{\alpha}\left(X_{\beta}\left(B_{\mu \gamma}\right)\right)+g\left(A_{E}^{*} X_{\gamma}, R\left(X_{\alpha}, X_{\mu}\right) X_{\beta}\right) \\
& +g\left(A_{E}^{*} X_{\beta}, R\left(X_{\alpha}, X_{\mu}\right) X_{\gamma}\right) .
\end{aligned}
$$

Next, as $\bar{R}=0$, from the Gauss-Codazzi relation (2.10), we get

$$
g(R(X, Y) Z, P W)=B(Y, Z) C(X, P W)-B(X, Z) C(Y, P W),
$$

for any $X, Y, Z, W \in \Gamma(T M)$. Applying (4.33) to (4.32), reduces it to

$$
\begin{aligned}
X_{\mu}\left(X_{\gamma}\left(B_{\alpha \beta}\right)\right) & =X_{\alpha}\left(X_{\beta}\left(B_{\mu \gamma}\right)\right)+B_{\mu \beta} C\left(X_{\alpha}, A_{E}^{*} X_{\gamma}\right) \\
& -B_{\alpha \beta} C\left(X_{\mu}, A_{E}^{*} X_{\gamma}\right)+B_{\mu \gamma} C\left(X_{\alpha}, A_{E}^{*} X_{\beta}\right) \\
& -B_{\alpha \gamma} C\left(X_{\mu}, A_{E}^{*} X_{\beta}\right) .
\end{aligned}
$$

Since we are working locally around a point $p \in M$, then $\tau\left(\left[X_{\alpha}, X_{\beta}\right]\right)=0$. Putting this into account, the assumption $\tau\left(X_{\alpha}\right)=0$, for all $\alpha \in\{1, \ldots, n\}$, and $\bar{R}=0$, we have from the Gauss-Codazzi equation (3.12) of $[7$, p. 95] that

$$
C\left(X_{\alpha}, A_{E}^{*} X_{\gamma}\right)=C\left(X_{\gamma}, A_{E}^{*} X_{\alpha}\right)
$$


Finally, placing (4.35) in (4.34) and taking trace with respect to $\mu$ and $\gamma$, we obtain the desired result and the proof ends here.

Theorem 4.6. Let $(M, g)$ be a screen integrable null hypersurface of a Lorentzian manifold $(\bar{M}, \bar{g})$. Under null MCF, the squared norm $\left|A_{E}^{*}\right|_{s}^{2}$ of the screen shape operator $A_{E}^{*}$ evolves according to the following

$$
\frac{\partial\left|A_{E}^{*}\right|_{s}^{2}}{\partial t}=\Delta^{s}\left|A_{E}^{*}\right|_{s}^{2}+2 \mathcal{H}\left|A_{E}^{*}\right|_{s}^{2}-2\left|\nabla^{s} B\right|_{s}^{2}
$$

where $\mathcal{H}:=\operatorname{tr}^{s}\left(A_{E}^{*} \circ A_{N}\right)$.

Proof. Considering $g^{\alpha \beta}(p)=\delta_{\alpha \beta}$, we have $\left|A_{E}^{*}\right|_{s}^{2}=g^{\alpha \mu} g^{\gamma \beta} B_{\alpha \mu} B_{\gamma \beta}=B_{\alpha \beta} B_{\alpha \beta}$. Using this relation and Proposition 4.5 we have

$$
\begin{aligned}
& \Delta^{s}\left|A_{E}^{*}\right|_{s}^{2}=2 B_{\alpha \beta} \Delta^{s} B_{\alpha \beta}+2\left|\nabla^{s} B\right|_{s}^{2} \\
& =2 B_{\alpha \beta} \operatorname{Hess}^{s}(\mathcal{S})-2 \mathcal{H}\left|A_{E}^{*}\right|_{s}^{2}+2\left|\nabla^{s} B\right|_{s}^{2}+2 \mathcal{S} \operatorname{tr}^{s}\left(A_{E}^{* 2} \circ A_{N}\right) .
\end{aligned}
$$

On the other hand, we can compute the time derivative of $\left|A_{E}^{*}\right|_{s}^{2}$ from $\left|A_{E}^{*}\right|_{s}^{2}=g^{\alpha \beta} g^{\gamma \mu} B_{\alpha \gamma} B_{\beta \mu}$ and the evolution equations of $g$ and $B$ in Theorem 4.3 with $\tau=0$ on the screen distribution as follows;

$$
\begin{aligned}
\frac{\partial\left|A_{E}^{*}\right|_{s}^{2}}{\partial t} & =2 \mathcal{S} C^{\alpha \beta} g^{\gamma \mu} B_{\alpha \gamma} B_{\beta \mu}+2 \mathcal{S} g^{\alpha \beta} C^{\gamma \mu} B_{\alpha \gamma} B_{\beta \mu} \\
& +g^{\alpha \beta} g^{\gamma \mu}\left\{\operatorname{Hess}^{s}(\mathcal{S})-\mathcal{S} B\left(X_{\alpha}, A_{N} X_{\gamma}\right)\right\} B_{\beta \mu} \\
& +g^{\alpha \beta} g^{\gamma \mu} B_{\alpha \gamma}\left\{\operatorname{Hess}^{s}(\mathcal{S})-\mathcal{S} B\left(X_{\beta}, A_{N} X_{\mu}\right)\right\} .
\end{aligned}
$$

Re-arranging some indices in (4.37), we get

$$
\begin{aligned}
\frac{\partial\left|A_{E}^{*}\right|_{s}^{2}}{\partial t} & =4 \mathcal{S} C^{\alpha \beta} g^{\gamma \mu} B_{\alpha \gamma} B_{\beta \mu}+2 g^{\alpha \beta} g^{\gamma \mu}\left\{\operatorname{Hess}^{s}(\mathcal{S})-\mathcal{S} B\left(X_{\alpha}, A_{N} X_{\gamma}\right)\right\} B_{\beta \mu} \\
& =4 \mathcal{S} \operatorname{tr}^{s}\left(A_{E}^{* 2} \circ A_{N}\right)+2 B_{\alpha \gamma} \operatorname{Hess}^{s}(\mathcal{S})-2 \mathcal{S} \operatorname{tr}^{s}\left(A_{E}^{* 2} \circ A_{N}\right) \\
& =2 \mathcal{S} \operatorname{tr}^{s}\left(A_{E}^{* 2} \circ A_{N}\right)+2 B_{\alpha \gamma} \operatorname{Hess}^{s}(\mathcal{S}) .
\end{aligned}
$$

Putting (4.36) and (4.38) we get the desired result and the proof ends here.

In order to discuss some geometric implications of the evolution equations in Theorems 4.3 and 4.6, we need the following well-known concept in null geometry.

A null hypersurface $(M, g)$ of a semi-Riemannian manifold $(\bar{M}, \bar{g})$ is called screen conformal $[7$, p. 51] if there exist a non-vanishing smooth function $\psi$ on a neighborhood $\mathcal{U}$ in $M$ such that $A_{N}=\psi A_{E}^{*}$, or equivalently,

$$
C(X, P Y)=\psi B(X, Y), \quad \forall X, Y \in \Gamma(T M) .
$$


We say that $M$ is screen homothetic if $\psi$ is a constant function on $M$.

Example 4.7. Consider the null cone in Example 4.4. By straightforward calculation, one gets $\bar{g}\left(\nabla_{E} X, E\right)=-\sum_{a=1}^{n+1} x_{a} X_{a}=0$, which implies that $\nabla_{E} X \in \Gamma\left(S\left(T \Lambda_{0}^{n+1}\right)\right)$. Hence, $A_{N} E=0$. Using Gauss-Codazzi equations, we calculate $C(X, Y)=\bar{g}\left(\nabla_{X} Y, N\right)=\bar{g}\left(\bar{\nabla}_{X} Y, N\right)=-\frac{1}{2 x_{0}^{2}} g(X, Y)$, for any $X, Y \in \Gamma\left(S\left(T \Lambda_{0}^{n+1}\right)\right)$. Consequently,

$$
A_{N} X=-\frac{1}{2 x_{0}^{2}} P X, \quad \forall X, Y \in \Gamma\left(S\left(T \Lambda_{0}^{n+1}\right)\right) .
$$

Considering (4.24) and (4.40) we deduce that

$$
A_{N} X=\frac{1}{2 x_{0}^{2}} A_{E}^{*} X, \quad \forall X \in \Gamma\left(S\left(T \Lambda_{0}^{n+1}\right)\right) .
$$

Hence, $\Lambda_{0}^{n+1}$ is screen globally conformal null hypersurface of $\mathbb{R}_{1}^{n+2}$, with a positive conformal factor $\psi=\frac{1}{2 x_{0}^{2}}$ globally define on $\Lambda_{0}^{n+1}$.

Using the previous concept and Theorem 4.6 we state the following.

Corollary 4.8. Under the hypothesis of Theorem 4.6, if the initial null hypersurface $M_{0}$ is screen conformal and the 1-form $\tau$ vanishes on $S\left(T M_{0}\right)$, then

$$
\frac{\partial\left|A_{E}^{*}\right|_{s}^{2}}{\partial t}=\Delta^{s}\left|A_{E}^{*}\right|_{s}^{2}+2 \psi\left|A_{E}^{*}\right|_{s}^{4}-2\left|\nabla^{s} B\right|_{s}^{2} .
$$

From the results of Corollary 4.8, we notice that the quadratic term in $\left|A_{E}^{*}\right|_{s}^{2}$ will cause finite time blow-up in the evolution equation of $\left|A_{E}^{*}\right|_{s}^{2}$. To understand the long term behavior of solutions near such blow-ups (or singularities) requires one to obtain a priori estimates. Such estimates can be integral or pointwise. In the latter case, the following maximum principle is always used.

Theorem $4.9([17])$. Assume that $g(t)$, for $t \in[0, T)$, is a family of Riemannian metrics on a manifold $M$, with a possible boundary $\partial M$, such that the dependence on $t$ is smooth. Let $u: M \times[0, T) \longrightarrow \mathbb{R}$ be a smooth function satisfying

$$
\frac{\partial u}{\partial t} \leq \Delta_{g(t)} u+\bar{g}(X(p, u, \nabla u, t), \nabla u)_{g(t)}+f(u),
$$

where $X$ and $f$ are respectively a continuous vector field and a locally Lipschitz function in their arguments. Then, suppose that for every $t \in[0, T)$ there exists a value $\delta>0$ and a compact subset $\mathcal{K} \subset M \backslash \partial M$ such that at every time $t^{\prime} \in(t-\delta, t+\delta) \cap\left[0, T^{\prime}\right)$ the maximum of $u\left(\cdot, t^{\prime}\right)$ is attained at 
least at one point of $\mathcal{K}$ (this is clearly true if $M$ is compact without boundary). Setting $u_{\max }(t):=\max _{p \in M} u(p, t)$ we have that the function $u_{\max }$ is locally Lipschitz, hence differentiable at almost every time $t \in[0, T)$ and at every differentiability time,

$$
\frac{d u_{\max }(t)}{d t} \leq f\left(u_{\max }(t)\right) .
$$

Consequently, if $v:\left[0, T^{\prime}\right) \longrightarrow \mathbb{R}$ is a solution of the ODE

$$
\frac{d v(t)}{d t}=f(v(t)), \quad v(0)=u_{\max }(0),
$$

for $T^{\prime} \leq T$, then $u \leq v$ in $M \times\left[0, T^{\prime}\right)$. Moreover, if $M$ is connected and at some time $\tilde{t} \in\left(0, T^{\prime}\right)$ we have $u_{\max }(\tilde{t})=v(\tilde{t})$, then $u=v$ in $M \times[0, \tilde{t}]$, that is, $u(\cdot, t)$ is constant in space.

Notice that there hold analogous results for the minimum of the solution of the opposite partial differential inequality. Moreover, the maximum principle for elliptic equations easily follows as the special case where all the quantities around do not depend on the time variable $t$. We will apply the above maximum principle to some of evolution equations derived earlier, when the initial null hypersurface $M_{0}$ is compact and mean convex. The hypersurface $M_{0}$ is mean convex if $\mathcal{S} \geq 0$ everywhere. It is well-known that mean convexity is preserved by the mean curvature flow [17].

Theorem 4.10. Let $M_{0}$ be a mean convex, screen conformal null hypersurface. If $\left|A_{E}^{*}\right|_{s}^{2}$ is not bounded as $t \longrightarrow T<+\infty$ during the null MCF of a compact null hypersurface, then it must satisfy the following lower bound for its blow-up rate:

$$
\max _{p \in M}\left|A_{E}^{*}\right|_{s}^{2}(p, t) \geq \frac{1}{2 \psi(T-t)},
$$

for every $t \in[0, T)$. Hence,

$$
\lim _{t \longrightarrow T} \max _{p \in M}\left|A_{E}^{*}\right|_{s}^{2}(p, t)=+\infty .
$$

Proof. From Corollary 4.8 and the maximum principle (Theorem 4.9), we deduce that

$$
\frac{\partial\left|A_{E}^{*}\right|_{s \max }^{2}}{\partial t} \leq 2 \psi\left|A_{E}^{*}\right|_{s \max }^{4}
$$

Notice that when the ambient space $\bar{M}$ is Lorentzian ian, we have that the screen distribution of $M_{0}$ is Riemannian. Consequently, $\left|A_{E}^{*}\right|_{s \max }^{2}$ is always positive otherwise we would get the case $A_{E}^{*}=0$ (that is; $M_{0}$ is totally 
geodesic in $\bar{M}$ ), rendering $M_{0}$ a hyperplane in $\bar{M}$, thereby contradicting the compactness assumption on $M_{0}$. More precisely, there are no compact hypersurfaces with zero mean curvatures [17]. Therefore, we can divide both sides by $\left|A_{E}^{*}\right|_{s \max }^{4}$ obtaining the following differential inequality for the locally Lipschitz function $\frac{1}{\left|A_{E}^{*}\right|_{s \max }^{2}}$, holding at almost every time $t \in[0, T)$,

$$
-\frac{d}{d t} \frac{1}{\left|A_{E}^{*}\right|_{s \max }^{2}} \leq 2 \psi
$$

Integration of the above inequality in the time interval $\left[t, t^{\prime}\right] \subset[0, T)$, we get

$$
\frac{1}{\left|A_{E}^{*}(\cdot, t)\right|_{s \max }^{2}}-\frac{1}{\left|A_{E}^{*}\left(\cdot, t^{\prime}\right)\right|_{s \max }^{2}} \leq 2 \psi\left(t^{\prime}-t\right) .
$$

Suppose that $A_{E}^{*}$ is not bounded in $[0, T)$, that is, there exists a sequence of times $t_{i}^{\prime} \longrightarrow T$ such that $\left|A_{E}^{*}\left(\cdot, t_{i}^{\prime}\right)\right|_{s \max }^{2} \longrightarrow+\infty$. Considering these times $t_{i}^{\prime}$ in the above inequality and sending $i \longrightarrow \infty$, we get

$$
\frac{1}{\left|A_{E}^{*}(\cdot, t)\right|_{s \max }^{2}} \leq 2 \psi(T-t)
$$

which completes the proof.

The above result shows that there will also be singularities in null MCF if the initial null hypersurface $M_{0}$ is screen conformal since the quantity $\left|A_{E}^{*}\right|_{s}^{2}$ blows-up in finite time. When this happens we say that $T$ is singular time for the null MCF. Moreover, we have the following.

Theorem 4.11. Given a compact, immersed screen conformal null hypersurface $M_{0}$ in $\bar{M}$ then there exists a unique null MCF defined on a maximal interval $\left[0, T_{\max }\right)$. Moreover, $T_{\max }$ is given by

$$
\max _{p \in M}\left|A_{E}^{*}\right|_{s}^{2} \geq \frac{1}{2 \psi\left(T_{\max }-t\right)}
$$

Let us turn to the evolution equation of $\mathcal{S}$. From Theorem 4.3 with $\tau=0$ on the screen distribution, we have

$$
\frac{\partial \mathcal{S}}{\partial t}=\Delta^{s} \mathcal{S}+\mathcal{S H}
$$

where $\mathcal{H}:=\operatorname{tr}^{s}\left(A_{E}^{*} \circ A_{N}\right)$.

Theorem 4.12. Let $M_{0}$ be a mean convex and compact screen conformal null hypersurface of $\bar{M}$. Under null MCF, the minimum $\mathcal{S}_{\min }$ of $\mathcal{S}$ is increasing, hence $\mathcal{S}$ is positive for every positive time. 
Proof. Consider the interval $\left(t_{0}, t_{1}\right) \subset \mathbb{R}^{+}$and suppose by contradiction that $\mathcal{S}_{\min }(t)<0$ and $\mathcal{S}_{\min }\left(t_{0}\right)=0$ for any $t$ in the above interval. Assume that $\left|A_{E}^{*}\right|_{s}^{2}$ is bounded on the interval $\left(t_{0}, t_{1}\right)$, that is $\left|A_{E}^{*}\right|_{s}^{2} \leq A$. Then, relation (4.45) and the maximum principle implies that

$$
\frac{\partial \mathcal{S}_{\min }}{\partial t} \geq \psi A \mathcal{S}_{\min }, \quad \forall t \in\left(t_{0}, t_{1}\right)
$$

Integrating (4.46) over $[s, t] \subset\left(t_{0}, t_{1}\right)$ we get

$$
\mathcal{S}_{\min }(t) \geq \exp \left(\int_{s}^{t} \psi A\right) \mathcal{S}_{\min }(s)
$$

Then, letting $s \longrightarrow t_{0}^{+}$in (4.47) gives $\mathcal{S}_{\min }(t) \geq 0$ for all $t \in\left(t_{0}, t_{1}\right)$ which is a contradiction. Next, let $k_{1}, \ldots, k_{n}$ be the eigenvalues of $A_{E}^{*}$ with respect to $\left\{X_{1}, \ldots, X_{n}\right\}$. It is easy to show that

$$
\left|A_{E}^{*}\right|_{s}^{2}-\frac{1}{n} \mathcal{S}^{2}=\frac{1}{n} \sum_{1 \leq \alpha \leq \beta \leq n}\left(k_{\beta}-k_{\alpha}\right)^{2},
$$

from which we deduce that $\left|A_{E}^{*}\right|_{s}^{2}-\frac{1}{n} \mathcal{S}^{2} \geq 0$. Using this inequality and (4.45) we get

$$
\frac{\partial \mathcal{S}}{\partial t}=\Delta \mathcal{S}+\mathcal{S H} \geq \Delta \mathcal{S}+\frac{\psi}{n} \mathcal{S}^{3}
$$

Let $u=-\mathcal{S}, X=0$ and $f(x)=\frac{\psi}{n} x^{3}$, then if $\mathcal{S}_{\min }(0)=0$, the ODE solution $v(t)=0$. Hence, if for some time $\mathcal{S}_{\min }(\tilde{t})=0$ we see that $\mathcal{S}_{\min }(\cdot, \tilde{t})$ is constant on $M_{t}$ and equal to zero. But there are no compact hypersurfaces with zero mean curvature (see [17]). Hence, $\mathcal{S}_{\text {min }}$ is always increasing during the flow and $\mathcal{S}$ is positive at every positive time.

Next, let us suppose that $M_{0}$ admits a symmetric induced Ricci tensor. Denote by $\tilde{R}$ the corresponding scalar curvature. As $\bar{R}=0$ we deduce, from (2.4.12) of [8, p. 69], that

$$
\tilde{R}=\operatorname{tr}^{s}\left(A_{E}^{*}\right) \operatorname{tr}^{s}\left(A_{N}\right)-\operatorname{tr}^{s}\left(A_{E}^{*} \circ A_{N}\right) .
$$

Theorem 4.13. If the scalar curvature $\tilde{R}$ is positive and bounded on a screen homothetic initial null hypersurface $M_{0}$ with $\psi>0$, then it remains bounded for all positive times $t$.

Proof. As $M_{0}$ is screen homothetic, (4.50) gives

$$
\tilde{R}=\psi \mathcal{S}^{2}-\psi\left|A_{E}^{*}\right|_{s}^{2}
$$


where $\psi$ is a constant function on $M_{0}$. Differentiating (4.51) and using the evolution equations of $\mathcal{S}$ and $\left|A_{E}^{*}\right|_{s}^{2}$ (see Theorems 4.3, 4.6 and Corollary 4.8), we get

$$
\begin{aligned}
\frac{\partial \tilde{R}}{\partial t} & =2 \psi \mathcal{S}\left(\Delta^{s} \mathcal{S}+\psi \mathcal{S}\left|A_{E}^{*}\right|_{s}^{2}\right)-\psi\left(\Delta^{s}\left|A_{E}^{*}\right|_{s}^{2}+2 \psi\left|A_{E}^{*}\right|_{s}^{4}-2\left|\nabla^{s} B\right|_{s}^{2}\right) \\
& =\Delta^{s}\left(\psi \mathcal{S}^{2}\right)-\Delta^{s}\left(\psi\left|A_{E}^{*}\right|_{s}^{2}\right)+2 \psi^{2} \mathcal{S}^{2}\left|A_{E}^{*}\right|_{s}^{2}-2 \psi^{2}\left|A_{E}^{*}\right|_{s}^{4} \\
& -2 \psi\left|\nabla^{s} \mathcal{S}\right|_{s}^{2}+2 \psi\left|\nabla^{s} B\right|_{s}^{2} \\
& =\Delta^{s} \tilde{R}+2 \psi\left|A_{E}^{*}\right|_{s}^{2} \tilde{R}+2 \psi\left(\left|\nabla^{s} B\right|_{s}^{2}-\left|\nabla^{s} \mathcal{S}\right|_{s}^{2}\right)
\end{aligned}
$$

Observe that the term $\left|\nabla^{s} B\right|_{s}^{2}-\left|\nabla^{s} \mathcal{S}\right|_{s}^{2}$ is non-positive and hence (4.52) gives

$$
\frac{\partial \tilde{R}}{\partial t} \leq \Delta^{s} \tilde{R}+2 \psi\left|A_{E}^{*}\right|_{s}^{2} \tilde{R} .
$$

Thus, taking $T^{\prime}<T$, if $\omega$ is the maximum of $\left|A_{E}^{*}\right|_{s}^{2}$ on $M \times\left[0, T^{\prime}\right]$, we have from (4.53) that $\frac{\partial \tilde{R}}{\partial t} \leq \Delta^{s} \tilde{R}+2 \psi \omega \tilde{R}$ on $M \times\left[0, T^{\prime}\right]$. By maximum principle (Theorem 4.9), we have $\frac{d \tilde{R}_{\max }}{d t} \leq 2 \psi \omega|\tilde{R}|_{\max }$, from which the result follows by integration and the arbitrariness of $T^{\prime}$. Hence, the proof ends here.

\section{Acknowledgments}

This work is based on the research supported wholly / in part by the National Research Foundation of South Africa (Grant Numbers: 95931 and 106072).

\section{References}

[1] A. Ashtekar and B. Krishnan, Dynamical horizons and their properties, Physical Review D, 68 (2003) 14030-10455.

[2] B. Andrews, Monotone quantities and unique limits for evolving convex hypersurfaces, Internat. Math. Res. Notices 1997 (1997), 1001-1031.

[3] C. Atindogbé, Scalar curvature on lightlike hypersurfaces, Balkan Society of Geometers, Geometry Balkan Press 2009, Applied Sciences, 11 (2009) 9-18.

[4] C. Atindogbé, J.-P. Ezin and J. Tossa, Pseudo-inversion of degenerate metrics Int. J. Math. Math. Sci., 55 (2003) 3479-3501.

[5] S. Aretakis, Lecture notes on general relativity, https://web.math.princeton.edu/ aretakis/columbiaGR.pdf.

[6] J. Dong and X. Liu, Totally Umbilical Lightlike Hypersurfaces in Robertson-Walker Spacetimes, Hindawi Publishing Corporation, 2014, Article ID 974695, pp. 10.

[7] K. L. Duggal and A. Bejancu. Lightlike submanifolds of semi-Riemannian manifolds and Applications. Mathematics and its Applications ( Kluwer Academic Publishers, 1996).

[8] K. L. Duggal and B. Sahin, Differential geometry of lightlike submanifolds (Frontiers in Mathematics, Birkhäuser Verlag, Basel, 2010). 
[9] L. Guanghan, T. Daping and W. Chuanxi, Translating solitons of mean curvature flow of noncompact submanifolds, Math Phys Anal Geom. 14 (2011) 83-99.

[10] M. Guti'errez and B. Olea. Induced Riemannian structures on null hypersurfaces. Math. Nachr. 289 (2016) 1219-1236.

[11] M. Gage, and R. Hamilton, The heat equation shrinking convex plane curves, J. Differential Geom. 23 no. 1 (1986) 69-96.

[12] V. Guillemin and A. Pollack, Differential Topology() Prentice Hall, 1974).

[13] G. Huisken, Asymptotic behaviour for singularities of the mean curvature flow, J. Diff. Geom. 31 (1990) 285-299 .

[14] G. Huisken, Flow by mean curvature of convex surfaces into spheres, J. Differential Geometry 20 (1984) 237-266.

[15] G. Huisken, Evolution of hypersurfaces by their curvature in Riemannian manifolds. Proceedings of the International Congress of Mathematicians, Doc. Math. 1998, Extra Vol. II, 349-360.

[16] D. H. Jin, Ascreen lightlike hypersurfaces of an indefinite Sasakian manifold. J. Korean Soc. Math. Educ. Ser. B: Pure Appl. Math., 20 no. 1 (2013) 25-35.

[17] C. Mantegazza, Lecture notes on mean curvature flow, Progress in Mathematics, 290 (2012).

[18] F. Massamba and S. Ssekajja, Quasi generalized CR-null submanifolds of indefinite nearly Sasakian manifolds, Arab. J. Math. 5 (2016) 87-101.

[19] B. O'Neill, Semi-Riemannian Geometry, with Applications to Relativity (New York: Academic Press 1983).

[20] D.N. Kupeli, Singular semi-Riemannian geometry, Mathematics and Its Applications (366, Kluwer Academic Publishers, 1996).

[21] K. Katsuno, Null hypersurfaces in Lorentzian manifold II, Math. Proc. Cambridge Philos. Soc. 89 (1981) 525-532.

[22] M. E. Taylor, Partial Differential Equations III: Nonlinear Equations, ( 117, Applied Mathematical Sciences, Springer, 1996).

1 School of Mathematics, Statistics and Computer Science

University of KwaZulu-Natal

Private Bag X01, Scottsville 3209

South Africa

E-mail address: ssekajja.samuel.buwaga@aims-senegal.org

2 School of Mathematics, Statistics and Computer Science

University of KwaZulu-Natal

Private Bag X01, Scottsville 3209

South Africa

E-mail address: massfort@yahoo.fr, Massamba@ukzn.ac.za 